\title{
BRINE CONTAMINATION OF GROUND WATER IN THE VICINITY OF THE BROOKHAVEN OIL FIELD, LINCOLN COUNTY, MISSISSIPPI
}

By Larry J. Slack, Charles G. O'Hara, and William T. Oakley

U.S. GEOLOGICAL SURVEY

Water-Resources Investigations Report 96-4023

Prepared in cooperation with the

U.S. ENVIRONMENTAL PROTECTION AGENCY

Jackson, Mississippi

1996 


\section{U.S. DEPARTMENT OF THE INTERIOR \\ BRUCE BABBITT, Secretary}

U.S. GEOLOGICAL SURVEY

Gordon P. Eaton, Director

For additional information write to:

District Chief

U.S. Geological Survey 308 South Airport Road Pearl, Mississippi 39208-6649
Copies of this report can be purchased from:

U.S. Geological Survey

Earth Science Information Center

Open-File Reports Section Box 25286, MS 517, Federal Center

Denver, Colorado 80225 


\section{CONTENTS}

Page

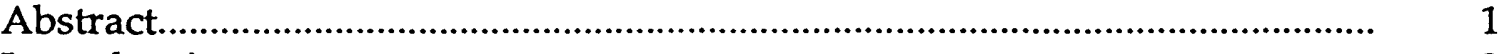

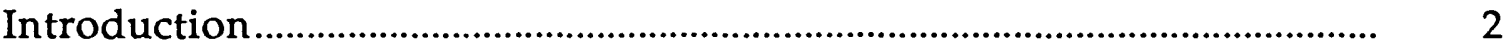

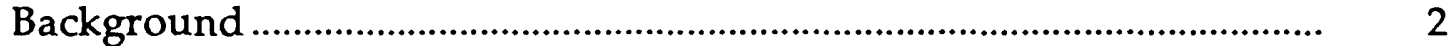

Purpose and scope...................................................................................... 3

Previous investigations........................................................................... 3

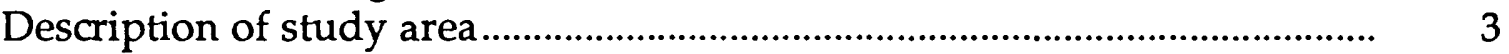

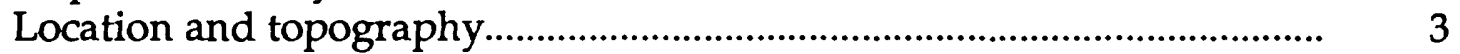

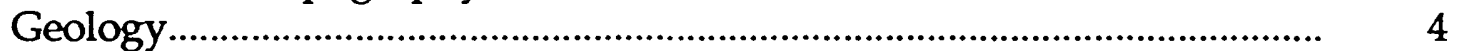

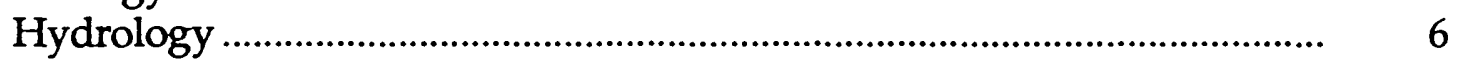

Citronelle aquifer ............................................................................ 6

Miocene aquifer system................................................................ 7

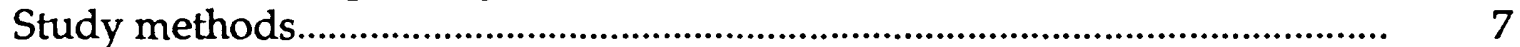

Chemical quality of brine and uncontaminated ground water.................. 13

Brine contamination of ground water.......................................................... 15

Brine contamination during August 1993-December 1994................... 15

Change in brine contamination from 1981-84 to $1993-94 \ldots \ldots \ldots \ldots \ldots \ldots \ldots \ldots . . . . . . . .22$

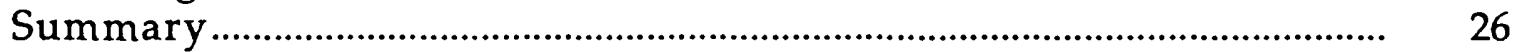

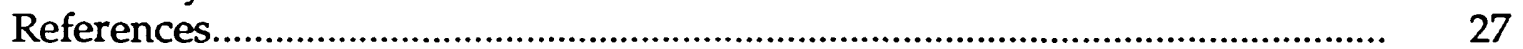

\section{ILLUSTRATIONS}

Figure 1. Map showing location of the study area, shallow wells (depth 120 feet or less), and trace of geologic sections, Lincoln County, Mississippi in pocket

2. Map showing location of deep wells (depth greater than 120 feet), Lincoln County, Mississippi in pocket

3. Map showing extent of brine contamination in shallow ground water (depth 120 feet or less) in Lincoln County, Mississippi (August 1993-December 1994) in pocket

4. Map showing extent of brine contamination in deep ground water (depth greater than 120 feet) in Lincoln County, Mississippi (August 1993-December 1994) .................in pocket

5. Generalized geologic sections $A-A^{\prime}$ and B-B' in the vicinity of the Brookhaven oil field in northwestern Lincoln County, Mississippi 


\section{TABLES}

Table 1. Site information for selected wells in Lincoln County,

2. Summary of analyses of brine samples from the Brookhaven oil field, Lincoln County, Mississippi

3. Summary of water-quality data for uncontaminated ground water in the Citronelle aquifer in northwestern Lincoln County, Mississippi

4. Water-quality data for selected wells in Lincoln County, Mississippi (August 1993-December 1994)

5. Summary of water-quality data for selected wells in Lincoln County, Mississippi (August 1993-December 1994)

6. Change in chloride concentrations in ground water in the vicinity of the Brookhaven oil field, Lincoln County, Mississippi (1981-84 to 1993-94) 
CONVERSION FACTORS AND WATER-QUALITY INFORMATION

\begin{tabular}{|c|c|c|}
\hline Multiply & By & To obtain \\
\hline barrel & 0.1590 & cubic meter \\
\hline cubic foot per second $\left(\mathrm{ft}^{3} / \mathrm{s}\right)$ & 0.02832 & cubic meter per second \\
\hline $\begin{array}{l}\text { cubic foot per day per square foot } \\
\text { times foot of aquifer thickness } \\
{\left[\left(\mathrm{ft}^{3} / \mathrm{d}\right) / \mathrm{ft}^{2}\right] \mathrm{ft}}\end{array}$ & 0.09290 & $\begin{array}{l}\text { cubic meter per day per } \\
\text { square meter times } \\
\text { meter of aquifer } \\
\text { thickness }\end{array}$ \\
\hline foot $(f t)$ & 0.3048 & meter \\
\hline foot per day $(\mathrm{ft} / \mathrm{d})$ & 0.3048 & meter per day \\
\hline gallon per day (gal/d) & 0.003785 & cubic meter per day \\
\hline $\begin{array}{l}\text { gallon per minute per foot } \\
{[(\mathrm{gal} / \mathrm{min}) / \mathrm{ft}]}\end{array}$ & 0.2070 & liter per second per meter \\
\hline inch (in.) & 25.4 & millimeter \\
\hline mile (mi) & 1.609 & kilometer \\
\hline square mile $\left(\mathrm{mi}^{2}\right)$ & 2.590 & square kilometer \\
\hline
\end{tabular}

To convert degrees Celsius $\left({ }^{\circ} \mathrm{C}\right)$ to degrees Fahrenheit $\left({ }^{\circ} \mathrm{F}\right)$, use the following:

$$
{ }^{\circ} \mathrm{C}=5 / 9\left({ }^{\circ} \mathrm{F}-32\right)
$$

Specific conductance is reported in microsiemens per centimeter at $25^{\circ} \mathrm{C}$ $(\mu \mathrm{S} / \mathrm{cm}) ; \mathrm{pH}$, in standard units. Chemical concentrations are given in milligrams per liter $(\mathrm{mg} / \mathrm{L})$ or micrograms per liter $(\mu \mathrm{g} / \mathrm{L})$. Milligrams and micrograms per liter are units expressing the weight of solute per volume of water. One thousand micrograms per liter is equivalent to $1 \mathrm{mg} / \mathrm{L}$. For concentrations less than $7,000 \mathrm{mg} / \mathrm{L}$, milligrams per liter are equivalent to parts per million. 


\title{
BRINE CONTAMINATION OF GROUND WATER IN THE VICINITY OF THE BROOKHAVEN OIL FIELD, LINCOLN COUNTY, MISSISSIPPI
}

\author{
by Larry J. Slack, Charles G. O'Hara, and William T. Oakley
}

\begin{abstract}
During 1993-94, chloride concentrations in ground water in the vicinity of the Brookhaven oil field in northwestern Lincoln County, Mississippi, ranged from 2.5 to $9,400 \mathrm{mg} / \mathrm{L}$ (milligrams per liter). Water from 89 (50 percent) of 177 wells was considered contaminated by brine--that is, had chloride concentrations greater than $20 \mathrm{mg} / \mathrm{L}$. However, the chloride concentrations in water from only 12 of the wells ( 7 percent) sampled during this study were greater than the recommended secondary limit for drinking water $(250 \mathrm{mg} / \mathrm{L})$.

From 1981-84 to 1993-94, the chloride concentration increased significantly (was greater than $20 \mathrm{mg} / \mathrm{L}$ and increased at least $2.0 \mathrm{mg} / \mathrm{L}$ ) at 23 of 81 sites. During the same period, the chloride concentration decreased significantly (was greater than $20 \mathrm{mg} / \mathrm{L}$ and decreased at least $2.0 \mathrm{mg} / \mathrm{L}$ ) at 20 sites. The largest chloride increase $(2,240 \mathrm{mg} / \mathrm{L})$ and decrease $(500 \mathrm{mg} / \mathrm{L})$ were at shallow wells, 45 and 120 feet deep, respectively.
\end{abstract}




\section{INTRODUCTION}

Large quantities of brine are withdrawn in conjunction with the production of petroleum. Since 1939, when petroleum production began in Mississippi, disposal of brine has resulted in brine contamination of ground water and streams. In the early years of oil production in Mississippi, brine was discharged onto the ground or into nearby streams. Later, in an effort to avoid surface-water contamination, producers pumped brine into evaporation ponds or pits. These disposal practices led to the brine contamination of shallow ground water. In 1978, these disposal methods were prohibited in the State (Mississippi State Oil and Gas Board, 1985). Most of the brine produced in the Brookhaven oil field is reinjected into the deep (greater than 4,000 ft) oil producing formations (Smith and others, 1988).

Information about the extent and the movement of the brine contamination and the constituents that the brine contains is useful to residents in the vicinity of the Brookhaven oil field who use ground water and to water-resource planners and managers. From August 1993 to December 1994, the U.S. Geological Survey (USGS), in cooperation with the U.S. Environmental Protection Agency (EPA), collected water-quality data as part of a basic research study to determine the extent of brine contamination in the vicinity of the Brookhaven oil field in southwestern Mississippi.

\section{Background}

The Brookhaven oil field is west of Brookhaven in northwestern Lincoln County, Mississippi (fig. 1) . Oil production in the field began on March 10, 1943. Annual production exceeded 1 million barrels by 1946 and reached a maximum of 5.25 million barrels in 1949. Annual production gradually decreased to less than 1 million barrels in 1973 and to less than 200,000 barrels in 1983. Annual production has averaged about 124,000 barrels for the last 10 years (1985-94) and has averaged only about 114,000 barrels for the last 5 years (1990-94). In 1994, production was about 111,000 barrels. Cumulative production of the Brookhaven oil field through 1994 was about 72,976,000 barrels. (All oil and brine production data are from the Mississippi State Oil and Gas Board annual reports published in 1944-95.)

During the initial development of oil reservoirs in the Brookhaven oil field, relatively little brine in proportion to oil was brought to the surface. For example, the brine-to-oil ratio was less than 0.5 until 1957. As oil is removed, formation water (brine) fills the pore spaces vacated by the oil, and subsequent pumping produces brine with the oil. Consequently, the brine-to-oil ratio increased to 1.0 by 1964 . In 1967, brine production reached a maximum of more than 6 million barrels and the brine-to-oil ratio increased to 5.6. By 1967, however, the brine-to-oil ratio decreased to about 1.5. For the last 10 years (1985-94), annual brine production has averaged about 550,000 barrels, and the brine-to-oil ratio has averaged about 4.4. For the last 5 years (1990-94), 
annual brine production has averaged about 444,000 barrels, and the brine-tooil ratio has averaged about 3.9. In 1994, brine production was about 385,000 barrels, and the brine-to-oil ratio was about 3.5. From 1943 to the end of 1994, cumulative brine production was about $60,561,000$ barrels, and the overall ratio of brine to oil produced was about 0.83 .

\section{Purpose and Scope}

This report defines the extent of brine contamination in shallow ground water in the vicinity of the Brookhaven oil field. The report is based largely on chemical analyses of water samples collected from selected wells during the period August 1993 through December 1994. The water-quality data are listed and summarized, maps showing the extent of brine contamination are presented, and the chloride data are compared with chloride data from a previous study by Kalkhoff (1986).

\section{Previous Investigations}

Previous investigations of the water quality in the vicinity of the Brookhaven oil field, Lincoln County, or the region were conducted by Newcome and Thomson (1970), and Kalkhoff $(1985,1986)$. Water-quality data for six samples collected between 1951 and 1968 from municipal water-supply wells completed in the Citronelle aquifer in Brookhaven were reported by Newcome and Thomson (1970), who also describe the general geology of Lincoln County. The geology and hydrology of the Citronelle aquifers statewide were described by Boswell (1979). Water-quality samples were collected in 1981 and 1982 from 10 wells in the Brookhaven oil field as part of a reconnaissance of major oil producing areas in Mississippi by Kalkhoff (1985). Lastly, Kalkhoff (1986) discussed in detail brine contamination of ground water and streams in the study area during the period from October 1983 to September 1984.

\section{DESCRIPTION OF THE STUDY AREA}

\section{Location and Topography}

The $100-\mathrm{mi}^{2}$ study area is located in northwestern Lincoln County, Mississippi (figs. 1-4). The Brookhaven oil field (approximately $15 \mathrm{mi}^{2}$ ) lies entirely within the study area. The study area is in the Pine Hills physiographic district, which Cross and Wales (1974) characterized as having a high and rolling land surface with moderately high ridges forming divides between streams. However, streams in the western and northern parts of the study area have eroded the land surface into narrow steep-sloped ridges (Kalkhoff, 1986). Land in the study area is used primarily for crops and pastures or is wooded; most homes are in the southern one-half of the study area (Perry Brumfield, Lincoln County Cooperative Extension Service Agent, 
oral commun., 1994). Part of the land in Brookhaven oil field is used for roads, well sites, storage tanks, pipelines, and other facilities related to oil production.

\section{Geology}

The geologic units that crop out or are present in the shallow subsurface in the study area consist of unconsolidated sediments of sand, clay, and gravel deposits which range in age from Miocene to Holocene (Newcome and Thomson, 1970). The geologic units, from oldest to youngest, are the undifferentiated Hattiesburg Formation of the Miocene Series, the Citronelle Formation of the Pliocene Series, and the Quaternary deposits that consist of loess and alluvium (Kalkhoff, 1986).

Sediments of the undifferentiated Hattiesburg Formation form the upper part of the Miocene Series in this area and are the oldest and deepest units investigated in this study (Newcome, 1975). Although these units consist mainly of silty clay with minor amounts of sand, some extensive sand units are present in these sediments in the study area (Kalkhoff, 1986). In this report, the undifferentiated Hattiesburg Formation is considered one unit and is included in the Miocene Series. The undifferentiated strata consist of several distinct sand layers separated by clay and silt.

Generalized geologic sections A-A' and B-B' in the study area (fig. 5) were prepared by Kalkhoff (1986) "from drillers logs supplied by water-well drillers and from electric [resistivity] logs made in oil test holes." Kalkhoff (1986) also prepared a third geologic section, C-C', which is not shown in this report but which was referred to in his description of the geology of the study area (p. 9):

Bicker (1969, p. 29) states that unweathered material from the Hattiesburg Formation contains clays that are gray, grayish brown, and blue. These weather rapidly to a greenish-gray color. On the basis of this description all sands deeper than the first reported blue or gray clay shown on drillers logs were assumed to be sands of the Hattiesburg Formation.

The Hattiesburg Formation in the study area consists of discontinuous sands separated by confining layers. The sands are fine to coarse grained and the confining layers are made up of silt and clay. Three sands are identified as separate units (layers A, B, and C) *** because the layers appear to be three discrete mapable units in the study area. They range in thickness from less than 10 to more than $90 \mathrm{ft}$ and generally dip to the south at approximately 20 to $30 \mathrm{ft} / \mathrm{mi}$. A westward dipping component to the sand layers is apparent as the corresponding sands in section B-B' are approximately $10 \mathrm{ft}$ higher in altitude than in section $C-C^{\prime}$. 

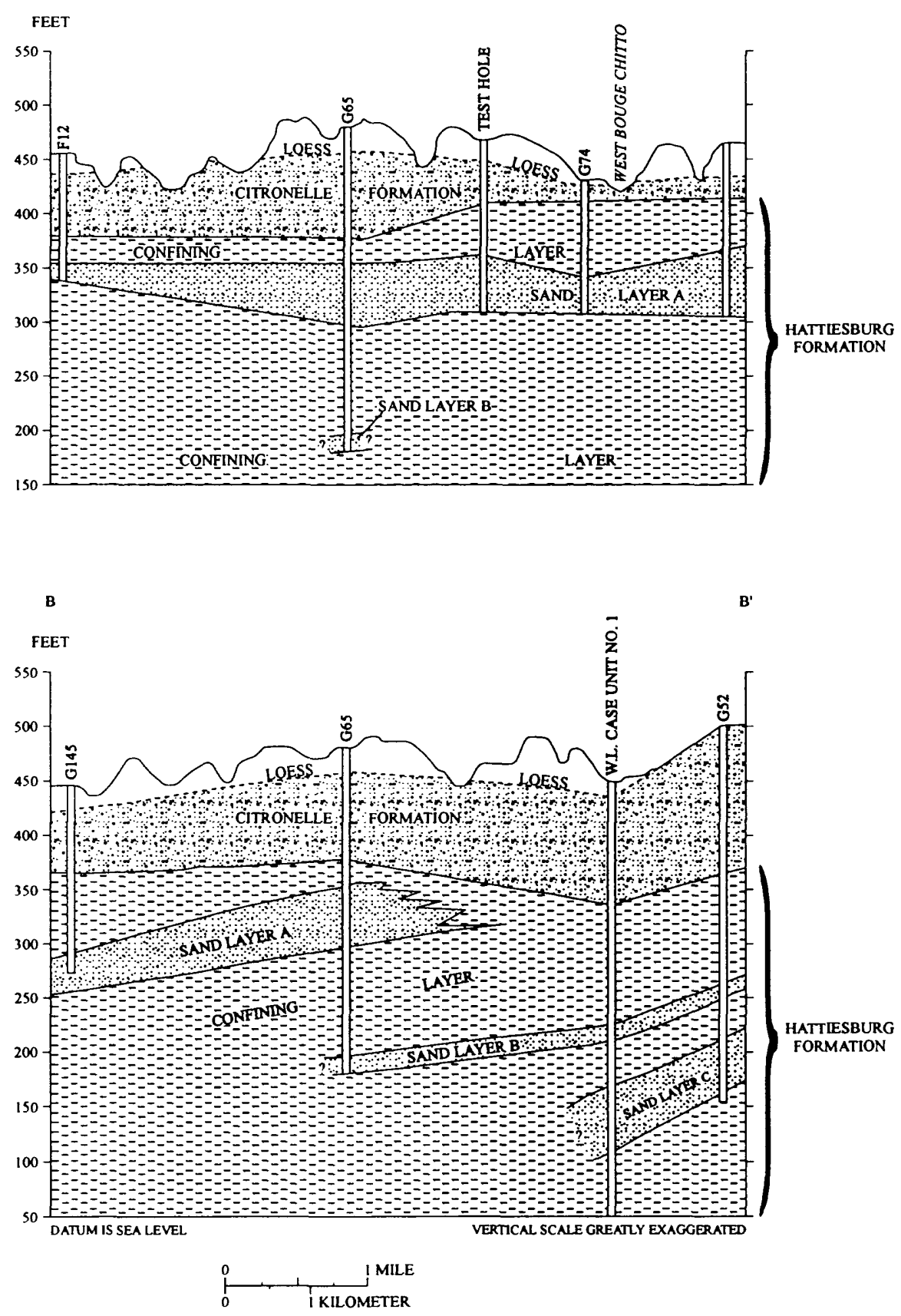

Figure 5. Generalized geologic sections $A-A^{\prime}$ and $B-B^{\prime}$ in the vicinity of the Brookhaven oil field in northwestern Lincoln County, Mississippi (from Kalkhoff, 1986). 
In describing the Citronelle Formation, Kalkhoff (1986, p. 12) stated the following:

Overlying the Hattiesburg Formation and exposed at places on the surface is the Citronelle Formation. The Citronelle Formation consists of discontinuous sand and gravel units separated by sandy clay lenses. Generally the thickest gravel layers are present in the basal part of the formation and the upper part is made up of sandy clay.

The base of the Citronelle ranges from an altitude of $440 \mathrm{ft}$ above sea level at the northern limit of the study area to an altitude of $370 \mathrm{ft}$ at the southern boundary. Through the study area, the base of the Citronelle dips at a rate of approximately $9.5 \mathrm{ft} / \mathrm{mi}$, slightly greater than the average regional dip of the formation reported by Boswell (1979). In the study area the Citronelle gradually thickens southward, ranging in thickness from approximately $40 \mathrm{ft}$ in the north to over $100 \mathrm{ft}$ in the central part of the oil field.

\section{Hydrology}

Two major sources of water in the study area are the Citronelle aquifer and the Miocene aquifer system. The Citronelle aquifer consists primarily of the sand and gravel units in the Citronelle Formation (Boswell, 1979) that contain sufficient saturated permeable material to yield significant quantities of water to wells. Similarly, the Miocene aquifer system consists primarily of the sand units in the Miocene Series (Newcome, 1975). The locations of wells for which water-quality data are used in this study are listed in table 1 and are shown in figures 1 and 3 (wells with depths of $120 \mathrm{ft}$ or less) and in figures 2 and 4 (wells with depths greater than $120 \mathrm{ft}$ ).

\section{Citronelle Aquifer}

Water in the Citronelle aquifer in the study area originates from precipitation that percolates through the unsaturated soil to the water table. Water in the aquifer moves laterally and discharges into nearby streams or moves downward into the underlying Miocene aquifer system. Water-level data for the Citronelle aquifer were not collected during the 1993-94 study. However, water-level measurements made in May 1984 indicate that the water-table surface of the Citronelle aquifer in the Brookhaven oil field slopes generally to the south and southwest (Kalkhoff, 1986). The direction and rate of water movement are dependent on lithologic and hydraulic characteristics of the aquifer. Few data on the hydraulic properties of the Citronelle aquifer are available, but the results of aquifer tests on two shallow wells at Brookhaven indicate that the transmissivity of the aquifer is about 4,000 to $5,300\left[\left(\mathrm{ft}^{3} / \mathrm{d}\right) / \mathrm{ft}^{2}\right] \mathrm{ft}$. [The term $\left[\left(\mathrm{ft}^{3} / \mathrm{d}\right) / \mathrm{ft}^{2}\right] \mathrm{ft}$ is hereafter reduced to $\mathrm{ft}^{2} / \mathrm{d}$, or feet 
squared per day.] The hydraulic conductivity is about 82 to $200 \mathrm{ft} / \mathrm{d}$ (Slack and Darden, 1991).

\section{Miocene Aquifer System}

Water in the shallowest parts of the Miocene aquifer system originates as infiltration from the overlying Citronelle aquifer, as infiltration from terrace and alluvial deposits in the valleys outside the study area, and as direct infiltration from rainfall where Miocene sediments crop out. The potentiometric surface of sand layer $\mathrm{A}$ in the Hattiesburg Formation in the southern part of the study area, based on limited (nine) water-level measurements in May 1984, slopes generally to the south (Kalkhoff, 1986). Kalkhoff reported that the potentiometric surface of sand layer $A$ "and the water-table surface of the Citronelle aquifer are at the same altitude" in the northern part of the study area. The similar potentiometric surfaces "may indicate that the two aquifers are hydraulically connected in this area." Southward, however, the potentiometric surface of sand layer A is lower than the water-table surface of the Citronelle aquifer, indicating the two aquifers are hydraulically separated.

Few data on the hydraulic properties of the Miocene aquifer system in the study area are available. However, the results of aquifer tests on three wells at Brookhaven, one well a few miles north of Brookhaven, and one well at Bogue Chitto (not shown) indicate that the transmissivity of the aquifer ranges from 620 to $7,200 \mathrm{ft}^{2} / \mathrm{d}$ (median transmissivity, 5,700 $\mathrm{ft}^{2} / \mathrm{d}$ ), and the hydraulic conductivity of the aquifer ranges from 20 to $140 \mathrm{ft} / \mathrm{d}$ (median conductivity, $71 \mathrm{ft} / \mathrm{d}$ ) (Slack and Darden, 1991).

\section{STUDY METHODS}

Water samples were collected and analyzed to identify oil-field-brine contamination in shallow ground water in the vicinity of the Brookhaven oil field. First preference in site selection was wells included in the 1983-84 study by Kalkhoff (1986). Secondary preference was wells currently in use or wells recently in use. Analyses of water analyses collected during this study were compared to water-quality data collected during previous investigations.

Water samples were collected and analyzed from 177 wells (table 1; figs. 1-4). Water from 141 wells (80 percent) is used for domestic purposes. Water from the remaining 36 wells ( 20 percent) is unused.

Water samples were collected and analyzed to determine concentrations of sodium, chloride, barium, and strontium, which often occur in relatively large concentrations in oil-field brines. To obtain samples that closely represent ground water from the water-bearing unit, most of the wells were pumped prior to sampling long enough to withdraw at least twice the volume of water in the casing. Water from 26 wells was collected with a point sampler which was opened at the bottom of a 2-in.-diameter test hole. The test holes were punched by the Corps of Engineers using a cone 
Table 1. Site information for selected wells in Lincoln County, Mississippi [CRNL, Citronelle aquifer; MOCN, Miocene aquifer system]

\begin{tabular}{|c|c|c|c|c|c|c|}
\hline $\begin{array}{l}\text { Site } \\
\text { number }\end{array}$ & $\begin{array}{l}\text { Local } \\
\text { number }\end{array}$ & $\begin{array}{l}\text { Well owner } \\
\text { or operator }\end{array}$ & Latitude & Longitude & $\begin{array}{l}\text { Depth } \\
\text { of well } \\
\text { (feet) }\end{array}$ & $\begin{array}{l}\text { Aquifer } \\
\text { unit code }\end{array}$ \\
\hline 1 & B37 & Ronald Durr & $31^{\circ} 37^{\prime} 31^{\prime \prime}$ & $90^{\circ} 37^{\prime} 00^{\prime \prime}$ & 152 & MOCN \\
\hline 2 & B48 & Raymond Bowman & $31^{\circ} 37^{\prime} 01^{\prime \prime}$ & $90^{\circ} 33^{\prime} 07^{\prime \prime}$ & 80 & CRNL \\
\hline 3 & $\mathrm{C} 17$ & CLDunn & $31^{\circ} 36^{\prime} 53^{\prime \prime}$ & $90^{\circ} 29^{\prime} 02^{\prime \prime}$ & 50 & CRNL \\
\hline 4 & $\mathrm{C} 18$ & Vicki Kinsey & $31^{\circ} 36^{\prime} 52^{\prime \prime}$ & $90^{\circ} 29^{\prime} 17^{\prime \prime}$ & 65 & CRNL \\
\hline 5 & $\mathrm{C} 19$ & T Smith & $31^{\circ} 37^{\prime} 22^{\prime \prime}$ & $90^{\circ} 29^{\prime} 58^{\prime \prime}$ & 63 & CRNL \\
\hline 6 & $\mathrm{C} 20$ & Odee Smith & $31^{\circ} 37^{\prime} 55^{\prime \prime}$ & $90^{\circ} 30^{\prime} 33^{\prime \prime}$ & 100 & CRNL \\
\hline 7 & $\mathrm{C} 21$ & Zeanni Hunt & $31^{\circ} 38^{\prime} 58^{\prime \prime}$ & $90^{\circ} 31^{\prime} 14^{\prime \prime}$ & 40 & CRNL \\
\hline 8 & $\mathrm{C} 22$ & J W Hunter & $31^{\circ} 38^{\prime} 50^{\prime \prime}$ & $90^{\circ} 31^{\prime} 25^{\prime \prime}$ & 200 & MOCN \\
\hline 9 & $\mathrm{C} 24$ & James E Smith & $31^{\circ} 38^{\prime} 27^{\prime \prime}$ & $90^{\circ} 30^{\prime} 33^{\prime \prime}$ & 49 & CRNL \\
\hline 10 & $\mathrm{C} 25$ & Claude Britt & $31^{\circ} 37^{\prime} 48^{\prime \prime}$ & $90^{\circ} 30^{\prime} 05^{\prime \prime}$ & 60 & CRNL \\
\hline 11 & $\mathrm{C} 28$ & Elmer Cockeram & $31^{\circ} 38^{\prime} 24^{\prime \prime}$ & $90^{\circ} 32^{\prime} 45^{\prime \prime}$ & 200 & MOCN \\
\hline 12 & $\mathrm{C} 29$ & Neil Lewis & $31^{\circ} 37^{\prime} 05^{\prime \prime}$ & $90^{\circ} 30^{\prime} 02^{\prime \prime}$ & 80 & CRNL \\
\hline 13 & C31 & Herman Smith & $31^{\circ} 37^{\prime} 16^{\prime \prime}$ & $90^{\circ} 30^{\prime} 11^{\prime \prime}$ & 60 & CRNL \\
\hline 14 & $\mathrm{C} 32$ & Kirby Humphreys & $31^{\circ} 37^{\prime} 06^{\prime \prime}$ & $90^{\circ} 31^{\prime} 22^{\prime \prime}$ & 100 & CRNL \\
\hline 15 & C34 & Evonne Maxwell & $31^{\circ} 37^{\prime} 09^{\prime \prime}$ & $90^{\circ} 31^{\prime} 37^{\prime \prime}$ & 100 & CRNL \\
\hline 16 & C35 & Jerry Bullock & $31^{\circ} 37^{\prime} 14^{\prime \prime}$ & $90^{\circ} 30^{\prime} 15^{\prime \prime}$ & 70 & CRNL \\
\hline 17 & $\mathrm{C} 36$ & Ed B Smith & $31^{\circ} 37^{\prime} 00^{\prime \prime}$ & $90^{\circ} 30^{\prime} 44^{\prime \prime}$ & 30 & CRNL \\
\hline 18 & C37 & Ricky Smith & $31^{\circ} 37^{\prime} 09^{\prime \prime}$ & $90^{\circ} 30^{\prime} 09^{\prime \prime}$ & 75 & CRNL \\
\hline 19 & $\mathrm{C} 45$ & Clarence Allen & $31^{\circ} 37^{\prime} 33^{\prime \prime}$ & $90^{\circ} 32^{\prime} 20^{\prime \prime}$ & 28 & CRNL \\
\hline 20 & $\mathrm{C} 49$ & J B Smith & $31^{\circ} 37^{\prime} 18^{\prime \prime}$ & $90^{\circ} 30^{\prime} 06^{\prime \prime}$ & 87 & CRNL \\
\hline 21 & C51 & J R Leming & $31^{\circ} 37^{\prime} 32^{\prime \prime}$ & $90^{\circ} 29^{\prime} 27^{\prime \prime}$ & 115 & MOCN \\
\hline 22 & $\mathrm{C} 52$ & Wayne Boyd & $31^{\circ} 38^{\prime} 08^{\prime \prime}$ & $90^{\circ} 30^{\prime} 38^{\prime \prime}$ & 61 & CRNL \\
\hline 23 & $\mathrm{C} 53$ & Howard Williams & $31^{\circ} 38^{\circ} 46^{\prime \prime}$ & $90^{\circ} 27^{\prime} 03^{\prime \prime}$ & 90 & CRNL \\
\hline 24 & C55 & Benjie Smith & $31^{\circ} 37^{\prime} 12^{\prime \prime}$ & $90^{\circ} 30^{\prime} 05^{\prime \prime}$ & 87 & CRNL \\
\hline 25 & C58 & Ford Crane & $31^{\circ} 38^{\prime} 26^{\prime \prime}$ & $90^{\circ} 28^{\prime} 02^{\prime \prime}$ & 64 & CRNL \\
\hline 26 & C61 & Henry Wallace & $31^{\circ} 36^{\prime} 44^{\prime \prime}$ & $90^{\circ} 26^{\prime} 53^{\prime \prime}$ & 89 & CRNL \\
\hline 27 & C62 & Roland Ross & $31^{\circ} 37^{\prime} 27^{n}$ & $90^{\circ} 27^{\prime} 48^{\prime \prime}$ & 60 & CRNL \\
\hline 28 & F20 & Jack B Smith & $31^{\circ} 35^{\prime} 50^{\prime \prime}$ & $90^{\circ} 33^{\prime} 42^{\prime \prime}$ & 85 & MOCN \\
\hline 29 & F21 & Johnie Watts & $31^{\circ} 33^{\prime} 19^{\prime \prime}$ & $90^{\circ} 33^{\prime} 11^{\prime \prime}$ & 80 & CRNL \\
\hline 30 & F24 & R H Williams & $31^{\circ} 36^{\prime} 29^{\prime \prime}$ & $90^{\circ} 33^{\prime} 10^{\prime \prime}$ & 87 & CRNL \\
\hline 31 & F27 & Percy Wilson & $31^{\circ} 34^{\prime} 19^{\prime \prime}$ & $90^{\circ} 33^{\prime} 15^{\prime \prime}$ & 98 & CRNL \\
\hline 32 & F28 & Wessie C Wiltch & $31^{\circ} 34^{\prime} 18^{\prime \prime}$ & $90^{\circ} 33^{\prime} 17^{\prime \prime}$ & 60 & CRNL \\
\hline 33 & F38 & Jaudon Smith & $31^{\circ} 32^{\prime} 58^{\prime \prime}$ & $90^{\circ} 34^{\prime} 34^{\prime \prime}$ & 72 & CRNL \\
\hline 34 & F39 & Ruth Watts & $31^{\circ} 32^{\prime} 05^{\prime \prime}$ & $90^{\circ} 35^{\prime} 39^{\prime \prime}$ & 80 & CRNL \\
\hline 35 & $\mathrm{~F} 40$ & Steve Case & $31^{\circ} 31^{\prime} 27^{\prime \prime}$ & $90^{\circ} 33^{\prime} 37^{\prime \prime}$ & 120 & CRNL \\
\hline
\end{tabular}


Table 1. Site information for selected wells in Lincoln County, Mississippi-Continued

\begin{tabular}{|c|c|c|c|c|c|c|}
\hline $\begin{array}{l}\text { Site } \\
\text { number }\end{array}$ & $\begin{array}{l}\text { Local } \\
\text { number }\end{array}$ & $\begin{array}{l}\text { Well owner } \\
\text { or operator }\end{array}$ & Latitude & Longitude & $\begin{array}{c}\text { Depth } \\
\text { of well } \\
\text { (feet) }\end{array}$ & $\begin{array}{l}\text { Aquifer } \\
\text { unit code }\end{array}$ \\
\hline 36 & F41 & Paul B Smith & $31^{\circ} 35^{\prime} 04^{\prime \prime}$ & $90^{\circ} 34^{\prime} 42^{\prime \prime}$ & 80 & CRNL \\
\hline 37 & F44 & Dykes A Britt & $31^{\circ} 35^{\prime} 48^{\prime \prime}$ & $90^{\circ} 32^{\prime} 56^{\prime \prime}$ & 70 & CRNL \\
\hline 38 & F48 & Annie B Case & $31^{\circ} 36^{\prime} 14^{\prime \prime}$ & $90^{\circ} 35^{\prime} 14^{\prime \prime}$ & 67 & CRNL \\
\hline 39 & G25 & Bryant Johnston & $31^{\circ} 36^{\prime} 20^{\prime \prime}$ & $90^{\circ} 29^{\prime} 56^{\prime \prime}$ & 150 & $\mathrm{MOCN}$ \\
\hline 40 & G.57 & Steve McFadden & $31^{\circ} 33^{\prime} 52^{\prime \prime}$ & $90^{\circ} 31^{\prime} 39^{\prime \prime}$ & 91 & CRNL \\
\hline 41 & G58 & Harry Case & $31^{\circ} 35^{\prime} 12^{\prime \prime}$ & $90^{\circ} 31^{\prime} 47^{\prime \prime}$ & 80 & CRNL \\
\hline 42 & G59 & Elvin Smith & $31^{\circ} 35^{\prime} 23^{\prime \prime}$ & $90^{\circ} 31^{\prime} 16^{\prime \prime}$ & 76 & CRNL \\
\hline 43 & G60 & Cecil Case & $31^{\circ} 35^{\prime} 36^{\prime \prime}$ & $90^{\circ} 32^{\prime} 03^{\prime \prime}$ & 100 & CRNL \\
\hline 44 & G61 & Robert Falvey & $31^{\circ} 35^{\prime} 42^{\prime \prime}$ & $90^{\circ} 31^{\prime} 52^{n}$ & 85 & CRNL \\
\hline 45 & G62 & Robert Falvey & $31^{\circ} 35^{\prime} 41^{\prime \prime}$ & $90^{\circ} 31^{\prime} 53^{\prime \prime}$ & 45 & CRNL \\
\hline 46 & G63 & Carol A Watts & $31^{\circ} 34^{\prime} 32^{\prime \prime}$ & $90^{\circ} 31^{\prime} 27^{\prime \prime}$ & 65 & CRNL \\
\hline 47 & G66 & Betty Warren & $31^{\circ} 36^{\prime} 18^{\prime \prime}$ & $90^{\circ} 32^{\prime} 10^{\prime \prime}$ & 48 & CRNL \\
\hline 48 & G67 & H R Owens & $31^{\circ} 34^{\prime} 34^{\prime \prime}$ & $90^{\circ} 31^{\prime} 55^{\prime \prime}$ & 150 & MOCN \\
\hline 49 & G68 & Aaron Achord & $31^{\circ} 33^{\prime} 45^{\prime \prime}$ & $90^{\circ} 31^{\prime} 22^{\prime \prime}$ & 308 & MOCN \\
\hline 50 & G69 & David Ballard & $31^{\circ} 35^{\prime} 12^{\prime \prime}$ & $90^{\circ} 31^{\prime} 51^{\prime \prime}$ & 85 & CRNL \\
\hline 51 & G71 & Rayburn Bowman & $31^{\circ} 36^{\prime} 23^{\prime \prime}$ & $90^{\circ} 32^{\prime} 11^{\prime \prime}$ & 256 & MOCN \\
\hline 52 & G72 & Dale Smith & $31^{\circ} 36^{\prime} 29^{\prime \prime}$ & $90^{\circ} 29^{\prime} 22^{\prime \prime}$ & 80 & CRNL \\
\hline 53 & G73 & S W Lee & $31^{\circ} 36^{\prime} 21^{n}$ & $90^{\circ} 29^{\prime} 50^{\prime \prime}$ & 120 & CRNL \\
\hline 54 & G74 & Jack Hostetler & $31^{\circ} 33^{\prime} 35^{\prime \prime}$ & $90^{\circ} 29^{\prime} 56^{\prime \prime}$ & 120 & MOCN \\
\hline 55 & G75 & Gene Simmons & $31^{\circ} 33^{\prime} 38^{\prime \prime}$ & $90^{\circ} 29^{\prime} 55^{n}$ & 100 & MOCN \\
\hline 56 & G76 & V Segars & $31^{\circ} 36^{\prime} 14^{\prime \prime}$ & $90^{\circ} 29^{\prime} 43^{\prime \prime}$ & 100 & CRNL \\
\hline 57 & G77 & Gary Norton & $31^{\circ} 36^{\prime} 17^{\prime \prime}$ & $90^{\circ} 29^{\prime} 38^{\prime \prime}$ & 100 & CRNL \\
\hline 58 & G79 & Marvin L Case & $31^{\circ} 36^{\prime} 14^{\prime \prime}$ & $90^{\circ} 29^{\prime} 01^{\prime \prime}$ & 75 & CRNL \\
\hline 59 & G80 & Vivian Smith & $31^{\circ} 35^{\prime} 53^{\prime \prime}$ & $90^{\circ} 29^{\prime} 28^{\prime \prime}$ & 118 & CRNL \\
\hline 60 & G82 & Clyde Norton & $31^{\circ} 36^{\prime} 03^{\prime \prime}$ & $90^{\circ} 29^{\prime} 33^{\prime \prime}$ & 45 & CRNL \\
\hline 61 & G83 & Jimmy D Smith & $31^{\circ} 35^{\prime} 52^{\prime \prime}$ & $90^{\circ} 29^{\prime} 25^{\prime \prime}$ & 50 & CRNL \\
\hline 62 & G85 & Steven R Sartin & $31^{\circ} 36^{\prime} 28^{\prime \prime}$ & $90^{\circ} 31^{\prime} 01^{\prime \prime}$ & 100 & CRNL \\
\hline 63 & G86 & M R Smith & $31^{\circ} 36^{\prime} 25^{\prime \prime}$ & $90^{\circ} 31^{\prime} 09^{\prime \prime}$ & 65 & CRNL \\
\hline 64 & G87 & Jimmie S Reeves & $31^{\circ} 36^{\prime} 12^{\prime \prime}$ & $90^{\circ} 31^{\prime} 11^{\prime \prime}$ & 60 & CRNL \\
\hline 65 & G88 & Willis Smith & $31^{\circ} 35^{\prime} 58^{\prime \prime}$ & $90^{\circ} 31^{\prime} 15^{\prime \prime}$ & 80 & CRNL \\
\hline 66 & G89 & Charles E Maxwell & $31^{\circ} 36^{\prime} 29^{\prime \prime}$ & $90^{\circ} 30^{\prime} 39^{\prime \prime}$ & 50 & CRNL \\
\hline 67 & G91 & Robert Watts & $31^{\circ} 32^{\prime} 27^{\prime \prime}$ & $90^{\circ} 32^{\prime} 27^{\prime \prime}$ & 106 & CRNL \\
\hline 68 & G95 & Glen Thompson & $31^{\circ} 33^{\prime} 41^{\prime \prime}$ & $90^{\circ} 30^{\prime} 31^{\prime \prime}$ & 30 & CRNL \\
\hline 69 & G97 & David Wilcher & $31^{\circ} 33^{\prime} 45^{\prime \prime}$ & $90^{\circ} 32^{\prime} 21^{\prime \prime}$ & 175 & MOCN \\
\hline 70 & G99 & Doug Warren & $31^{\circ} 34^{\prime} 29^{\prime \prime}$ & $90^{\circ} 31^{\prime} 31^{\prime \prime}$ & 48 & CRNL \\
\hline
\end{tabular}


Table 1. Site information for selected wells in Lincoln County, Mississippi-Continued

\begin{tabular}{|c|c|c|c|c|c|c|}
\hline $\begin{array}{l}\text { Site } \\
\text { number }\end{array}$ & $\begin{array}{l}\text { Local } \\
\text { number }\end{array}$ & $\begin{array}{l}\text { Well owner } \\
\text { or operator }\end{array}$ & Latitude & Longitude & $\begin{array}{l}\text { Depth } \\
\text { of well } \\
\text { (feet) }\end{array}$ & $\begin{array}{l}\text { Aquifer } \\
\text { unit code }\end{array}$ \\
\hline 71 & $\mathrm{G} 100$ & Earl Case & $31^{\circ} 35^{\prime} 18^{\prime \prime}$ & $90^{\circ} 31^{\prime} 42^{\prime \prime}$ & 90 & CRNL \\
\hline 72 & G106 & Jesse Warren & $31^{\circ} 33^{\prime} 58^{\prime \prime}$ & $90^{\circ} 31^{\prime} 55^{\prime \prime}$ & 92 & CRNL \\
\hline 73 & G107 & Billy Reed & $31^{\circ} 34^{\prime} 46^{\prime \prime}$ & $90^{\circ} 32^{\prime} 07^{\prime \prime}$ & 120 & CRNL \\
\hline 74 & G109 & Willie C Case & $31^{\circ} 35^{\prime} 44^{\prime \prime}$ & $90^{\circ} 31^{\prime 2} 21^{\prime \prime}$ & 79 & CRNL \\
\hline 75 & G110 & Charles W Smith & $31^{\circ} 35^{\prime} 59^{\prime \prime}$ & $90^{\circ} 30^{\prime} 46^{\prime \prime}$ & 100 & CRNL \\
\hline 76 & G114 & Charles Case & $31^{\circ} 35^{\prime} 40^{\prime \prime}$ & $90^{\circ} 32^{\prime} 05^{\prime \prime}$ & 84 & CRNL \\
\hline 77 & G117 & W Buddy Case & $31^{\circ} 35^{\prime} 52^{\prime \prime}$ & $90^{\circ} 32^{\prime} 49^{\prime \prime}$ & 73 & CRNL \\
\hline 78 & G118 & Eugene Case & $31^{\circ} 34^{\prime} 08^{\prime \prime}$ & $90^{\circ} 31^{\prime} 54^{\prime \prime}$ & 69 & CRNL \\
\hline 79 & G119 & Hosie Smith & $31^{\circ} 35^{\prime} 45^{\prime \prime}$ & $90^{\circ} 31^{\prime} 27^{\prime \prime}$ & 98 & CRNL \\
\hline 80 & G120 & Randy Britt & $31^{\circ} 35^{\prime} 53^{\prime \prime}$ & $90^{\circ} 31^{\prime} 24^{\prime \prime}$ & 85 & CRNL \\
\hline 81 & G123 & Case Grocery & $31^{\circ} 35^{\prime} 23^{\prime \prime}$ & $90^{\circ} 31^{\prime} 09^{\prime \prime}$ & 75 & CRNL \\
\hline 82 & G127 & Francis M Case & $31^{\circ} 35^{\prime} 34^{\prime \prime}$ & $90^{\circ} 32^{\prime} 21^{\prime \prime}$ & 70 & CRNL \\
\hline 83 & G131 & John Pounds & $31^{\circ} 35^{\prime} 22^{\prime \prime}$ & $90^{\circ} 30^{\prime} 13^{\prime \prime}$ & 200 & MOCN \\
\hline 84 & G133 & Rosy Reed & $31^{\circ} 34^{\prime} 44^{\prime \prime}$ & $90^{\circ} 31^{\prime} 46^{\prime \prime}$ & 120 & CRNL \\
\hline 85 & G134 & Sherry Spencer & $31^{\circ} 34^{\prime} 53^{\prime \prime}$ & $90^{\circ} 31^{\prime} 55^{\prime \prime}$ & 50 & CRNL \\
\hline 86 & G135 & W C Little & $31^{\circ} 34^{\prime} 59^{\prime \prime}$ & $90^{\circ} 31^{\prime} 50^{\prime \prime}$ & 140 & MOCN \\
\hline 87 & G137 & Maurice L Case & $31^{\circ} 35^{\prime} 31^{\prime \prime}$ & $90^{\circ} 32^{\prime} 23^{\prime \prime}$ & 75 & CRNL \\
\hline 88 & G138 & Dallas Anding & $31^{\circ} 35^{\prime} 31^{\prime \prime}$ & $90^{\circ} 32^{\prime} 44^{\prime \prime}$ & 85 & CRNL \\
\hline 89 & G139 & Curtis Nations & $31^{\circ} 35^{\prime} 35^{\prime \prime}$ & $90^{\circ} 32^{\prime} 25^{\prime \prime}$ & 93 & CRNL \\
\hline 90 & G140 & Winnie Smith & $31^{\circ} 35^{\prime} 39^{\prime \prime}$ & $90^{\circ} 32^{\prime} 44^{\prime \prime}$ & 80 & CRNL \\
\hline 91 & G142 & T Banks & $31^{\circ} 34^{\prime} 37^{\prime \prime}$ & $90^{\circ} 29^{\prime} 41^{\prime \prime}$ & 40 & CRNL \\
\hline 92 & G143 & Harold Case & $31^{\circ} 33^{\prime} 40^{\prime \prime}$ & $90^{\circ} 31^{\prime} 57^{\prime \prime}$ & 80 & CRNL \\
\hline 93 & G144 & Paul M Lewis & $31^{\circ} 33^{\prime} 36^{\prime \prime}$ & $90^{\circ} 30^{\prime} 59^{\prime \prime}$ & 75 & CRNL \\
\hline 94 & G148 & W B Eastley & $31^{\circ} 32^{\prime} 40^{\prime \prime}$ & $90^{\circ} 29^{\prime} 42^{\prime \prime}$ & 68 & CRNL \\
\hline 95 & G151 & Charlie Middleton & $31^{\circ} 34^{\prime} 49^{\prime \prime}$ & $90^{\circ} 29^{\prime} 13^{\prime \prime}$ & 180 & MOCN \\
\hline 96 & G152 & Mary Manning & $31^{\circ} 33^{\prime} 37^{\prime \prime}$ & $90^{\circ} 30^{\prime} 50^{\prime \prime}$ & 165 & MOCN \\
\hline 97 & G161 & John W Smith & $31^{\circ} 33^{\prime} 34^{\prime \prime}$ & $90^{\circ} 30^{\prime} 13^{\prime \prime}$ & 62 & CRNL \\
\hline 98 & G162 & Bolivar Coke & $31^{\circ} 34^{\prime} 33^{\prime \prime}$ & $90^{\circ} 31^{\prime} 52^{\prime \prime}$ & 128 & CRNL \\
\hline 99 & G163 & TS Webb & $31^{\circ} 34^{\prime} 25^{n}$ & $90^{\circ} 28^{\prime} 50^{\prime \prime}$ & 87 & CRNL \\
\hline 100 & G165 & D R Herndon & $31^{\circ} 35^{\prime} 03^{\prime \prime}$ & $90^{\circ} 30^{\prime} 09^{\prime \prime}$ & 114 & MOCN \\
\hline 101 & G169 & Donald McCullough & $31^{\circ} 36^{\prime} 25^{\prime \prime}$ & $90^{\circ} 30^{\prime} 24^{\prime \prime}$ & 115 & CRNL \\
\hline 102 & G175 & Stanley Smith & $31^{\circ} 32^{\prime} 17^{\prime \prime}$ & $90^{\circ} 29^{\prime} 33^{\prime \prime}$ & 70 & CRNL \\
\hline 103 & G177 & Herman Smith & $31^{\circ} 34^{\prime} 01^{\prime \prime}$ & $90^{\circ} 31^{\prime} 15^{\prime \prime}$ & 120 & CRNL \\
\hline 104 & G178 & Sam C Smith & $31^{\circ} 36^{\prime} 31^{\prime \prime}$ & $90^{\circ} 31^{\prime} 21^{\prime \prime}$ & 60 & CRNL \\
\hline 105 & G180 & Aaron Achord & $31^{\circ} 33^{\prime} 49^{\prime \prime}$ & $90^{\circ} 31^{\prime 22}$ & 147 & CRNL \\
\hline
\end{tabular}


Table 1. Site information for selected wells in Lincoln County, Mississippi-Continued

\begin{tabular}{|c|c|c|c|c|c|c|}
\hline $\begin{array}{c}\text { Site } \\
\text { number }\end{array}$ & $\begin{array}{l}\text { Local } \\
\text { number }\end{array}$ & $\begin{array}{l}\text { Well owner } \\
\text { or operator }\end{array}$ & Latitude & Longitude & $\begin{array}{l}\text { Depth } \\
\text { of well } \\
\text { (feet) }\end{array}$ & $\begin{array}{l}\text { Aquifer } \\
\text { unit code }\end{array}$ \\
\hline 106 & G181 & Bobby Moore & $31^{\circ} 34^{\prime} 01^{\prime \prime}$ & $90^{\circ} 31^{\prime} 09^{\prime \prime}$ & 150 & CRNL \\
\hline 107 & G182 & John Jordan & $31^{\circ} 33^{\prime} 56^{\prime \prime}$ & $90^{\circ} 31^{\prime} 19^{\prime \prime}$ & 148 & CRNL \\
\hline 108 & G186 & Buddy Price & $31^{\circ} 33^{\prime} 55^{\prime \prime}$ & $90^{\circ} 31^{\prime} 38^{\prime \prime}$ & 150 & MOCN \\
\hline 109 & G208 & V L Day & $31^{\circ} 33^{\prime} 44^{\prime \prime}$ & $90^{\circ} 31^{\prime 2} 23^{\prime \prime}$ & 160 & MOCN \\
\hline 110 & G209 & A C Lofton & $31^{\circ} 33^{\prime} 57^{\prime \prime}$ & $90^{\circ} 31^{\prime} 08^{\prime \prime}$ & 120 & MOCN \\
\hline 111 & G210 & A C Lofton & $31^{\circ} 33^{\prime} 57^{\prime \prime}$ & $90^{\circ} 31^{\prime} 08^{\prime \prime}$ & 65 & CRNL \\
\hline 112 & G211 & A C Lofton & $31^{\circ} 33^{\prime} 58^{\prime \prime}$ & $90^{\circ} 31^{\prime} 07^{\prime \prime}$ & 125 & MOCN \\
\hline 113 & $\mathrm{G} 212$ & Billy Reed & $31^{\circ} 34^{\prime} 47^{n}$ & $90^{\circ} 32^{\prime} 01^{\prime \prime}$ & 120 & CRNL \\
\hline 114 & $\mathrm{G} 213$ & Leland Smith & $31^{\circ} 33^{\prime} 49^{\prime \prime}$ & $90^{\circ} 32^{\prime} 02^{\prime \prime}$ & 80 & CRNL \\
\hline 115 & $\mathrm{G} 214$ & Zetus Grocery & $31^{\circ} 33^{\prime} 50^{\prime \prime}$ & $90^{\circ} 31^{\prime} 44^{\prime \prime}$ & 65 & CRNL \\
\hline 116 & G215 & Clem B Smith & $31^{\circ} 33^{\prime} 48^{\prime \prime}$ & $90^{\circ} 31^{\prime} 55^{\prime \prime}$ & 80 & CRNL \\
\hline 117 & G216 & Truly Smith & $31^{\circ} 35^{\prime} 44^{\prime \prime}$ & $90^{\circ} 31^{\prime} 02^{\prime \prime}$ & 68 & CRNL \\
\hline 118 & G217 & Charles Cupit & $31^{\circ} 33^{\prime} 41^{\prime \prime}$ & $90^{\circ} 30^{\prime} 51^{\prime \prime}$ & 60 & CRNL \\
\hline 119 & G218 & Kenneth Williams & $31^{\circ} 35^{\prime} 36^{\prime \prime}$ & $90^{\circ} 30^{\prime} 41^{\prime \prime}$ & 70 & CRNL \\
\hline 120 & $\mathrm{G} 247$ & Joel Smith & $31^{\circ} 34^{\prime} 25^{\prime \prime}$ & $90^{\circ} 28^{\prime} 20^{\prime \prime}$ & 160 & $\mathrm{MOCN}$ \\
\hline 121 & G258 & Andy Cupit & $31^{\circ} 33^{\prime} 57^{\prime \prime}$ & $90^{\circ} 31^{\prime} 31^{\prime \prime}$ & 145 & CRNL \\
\hline 122 & G262 & Miss Highway Dept & $31^{\circ} 32^{\prime} 45^{\prime \prime}$ & $90^{\circ} 27^{\prime} 53^{\prime \prime}$ & 160 & MOCN \\
\hline 123 & G267 & John Allgood & $31^{\circ} 33^{\prime} 43^{\prime \prime}$ & $90^{\circ} 29^{\prime} 30^{\prime \prime}$ & 113 & MOCN \\
\hline 124 & G268 & Glynn C Smith & $31^{\circ} 34^{\prime} 29^{\prime \prime}$ & $90^{\circ} 32^{\prime} 03^{\prime \prime}$ & 126 & CRNL \\
\hline 125 & $\mathrm{G} 271$ & Andrew Sisung & $31^{\circ} 35^{\prime} 28^{\prime \prime}$ & $90^{\circ} 31^{\prime} 56^{\prime \prime}$ & 66 & CRNL \\
\hline 126 & $\mathrm{G} 272$ & Tommy Crum & $31^{\circ} 33^{\prime} 52^{n}$ & $90^{\circ} 29^{\prime} 06^{\prime \prime}$ & 58 & CRNL \\
\hline 127 & G275 & Janice Thompson & $31^{\circ} 35^{\prime} 27^{\prime \prime}$ & $90^{\circ} 31^{\prime} 45^{\prime \prime}$ & 84 & CRNL \\
\hline 128 & G276 & Larry Warren & $31^{\circ} 33^{\prime} 54^{\prime \prime}$ & $90^{\circ} 32^{\prime} 07^{\prime \prime}$ & 90 & CRNL \\
\hline 129 & G279 & H S Woolley & $31^{\circ} 35^{\prime} 12^{\prime \prime}$ & $90^{\circ} 28^{\prime} 10^{\prime \prime}$ & 85 & CRNL \\
\hline 130 & G283 & Tony Lynn Smith & $31^{\circ} 32^{\prime} 46^{\prime \prime}$ & $90^{\circ} 32^{\prime} 50^{\prime \prime}$ & 78 & CRNL \\
\hline 131 & G312 & Larry McFadden & $31^{\circ} 33^{\prime} 52^{\prime \prime}$ & $90^{\circ} 31^{\prime} 36^{\prime \prime}$ & 93 & CRNL \\
\hline 132 & G316 & Randall Smith & $31^{\circ} 33^{\prime} 47^{\prime \prime}$ & $90^{\circ} 32^{\prime} 36^{\prime \prime}$ & 160 & CRNL \\
\hline 133 & G324 & Glynn C Smith & $31^{\circ} 34^{\prime} 27^{\prime \prime}$ & $90^{\circ} 31^{\prime} 57^{\prime \prime}$ & 120 & CRNL \\
\hline 134 & G333 & Dewayne Nevels & $31^{\circ} 33^{\prime} 26^{\prime \prime}$ & $90^{\circ} 30^{\prime} 13^{\prime \prime}$ & 160 & MOCN \\
\hline 135 & G335 & W G Sellers & $31^{\circ} 33^{\prime} 04^{\prime \prime}$ & $90^{\circ} 27^{\prime} 20^{\prime \prime}$ & 180 & MOCN \\
\hline 136 & G337 & Judy Patt & $31^{\circ} 33^{\prime} 55^{\prime \prime}$ & $90^{\circ} 31^{\prime} 57^{\prime \prime}$ & 170 & CRNL \\
\hline 137 & G346 & Clarence Hutson & $31^{\circ} 33^{\prime} 34^{\prime \prime}$ & $90^{\circ} 28^{\prime} 51^{\prime \prime}$ & 170 & MOCN \\
\hline 138 & G354 & Kenneth Smith & $31^{\circ} 36^{\prime} 08^{\prime \prime}$ & $90^{\circ} 31^{\prime} 06^{\prime \prime}$ & 89 & CRNL \\
\hline 139 & G356 & Richard Edwards & $31^{\circ} 35^{\prime} 02^{\prime \prime}$ & $90^{\circ} 32^{\prime} 04^{\prime \prime}$ & 107 & CRNL \\
\hline 140 & G365 & Margarite Smith & $31^{\circ} 34^{\prime} 30^{\prime \prime}$ & $90^{\circ} 31^{\prime} 40^{\prime \prime}$ & 86 & CRNL \\
\hline
\end{tabular}


Table 1. Site information for selected wells in Lincoln County, Mississippi-Continued

\begin{tabular}{|c|c|c|c|c|c|c|}
\hline $\begin{array}{l}\text { Site } \\
\text { number }\end{array}$ & $\begin{array}{l}\text { Local } \\
\text { number }\end{array}$ & $\begin{array}{l}\text { Well owner } \\
\text { or operator }\end{array}$ & Latitude & Longitude & $\begin{array}{c}\text { Depth } \\
\text { of well } \\
\text { (feet) }\end{array}$ & $\begin{array}{l}\text { Aquifer } \\
\text { unit code }\end{array}$ \\
\hline 141 & G368 & Bernice Smith & $31^{\circ} 35^{\prime} 25^{\prime \prime}$ & $90^{\circ} 31^{\prime} 33^{\prime \prime}$ & 85 & CRNL \\
\hline 142 & G370 & Bobby Reed & $31^{\circ} 34^{\prime} 58^{\prime \prime}$ & $90^{\circ} 31^{\prime} 52^{\prime \prime}$ & 86 & CRNL \\
\hline 143 & $\mathrm{G} 377$ & Ed Lambright & $31^{\circ} 33^{\prime} 33^{\prime \prime}$ & $90^{\circ} 30^{\prime} 16^{\prime \prime}$ & 160 & MOCN \\
\hline 144 & $\mathrm{G} 379$ & Donald Campbell & $31^{\circ} 33^{\prime} 54^{\prime \prime}$ & $90^{\circ} 28^{\prime} 50^{\prime \prime}$ & 160 & CRNL \\
\hline 145 & G380 & Betty Freeman & $31^{\circ} 36^{\prime} 18^{\prime \prime}$ & $90^{\circ} 32^{\prime} 11^{\prime \prime}$ & 165 & MOCN \\
\hline 146 & G381 & Roscoe Newton & $31^{\circ} 33^{\prime} 47^{\prime \prime}$ & $90^{\circ} 32^{\prime} 30^{\prime \prime}$ & 93 & CRNL \\
\hline 147 & G382 & Ed Lambright & $31^{\circ} 33^{\prime} 33^{\prime \prime}$ & $90^{\circ} 30^{\prime} 16^{\prime \prime}$ & 58 & CRNL \\
\hline 148 & G383 & Anna Littleton & $31^{\circ} 35^{\prime} 57^{\prime \prime}$ & $90^{\circ} 30^{\prime} 15^{\prime \prime}$ & 57 & CRNL \\
\hline 149 & G403 & Bailey Dunaway & $31^{\circ} 35^{\prime} 24^{\prime \prime}$ & $90^{\circ} 29^{\prime} 23^{\prime \prime}$ & 110 & CRNL \\
\hline 150 & G404 & Bailey Dunaway & $31^{\circ} 35^{\prime} 48^{\prime \prime}$ & $90^{\circ} 29^{\prime} 14^{\prime \prime}$ & 40 & CRNL \\
\hline 151 & K35 & John Newell & $31^{\circ} 30^{\prime} 58^{\prime \prime}$ & $90^{\circ} 34^{\prime} 50^{\prime \prime}$ & 80 & CRNL \\
\hline 152 & $\mathrm{C} 063$ & CР 27 & $31^{\circ} 37^{\prime} 12^{\prime \prime}$ & $90^{\circ} 28^{\prime} 22^{\prime \prime}$ & 30 & CRNL \\
\hline 153 & $\mathrm{C} 064$ & $\mathrm{CP} 4$ & $31^{\circ} 36^{\prime} 44^{\prime \prime}$ & $90^{\circ} 28^{\prime} 18^{\prime \prime}$ & 16 & CRNL \\
\hline 154 & $\mathrm{C} 065$ & CP 28 & $31^{\circ} 38^{\prime} 11^{\prime \prime}$ & $90^{\circ} 27^{\prime} 51^{\prime \prime}$ & 57 & CRNL \\
\hline 155 & $\mathrm{C} 066$ & CP 26 & $31^{\circ} 37^{\prime} 30^{\prime \prime}$ & $90^{\circ} 28^{\prime} 19^{\prime \prime}$ & 26 & CRNL \\
\hline 156 & $\mathrm{C} 067$ & CP 22 & $31^{\circ} 39^{\prime} 05^{\prime \prime}$ & $90^{\circ} 30^{\prime} 04^{\prime \prime}$ & 45 & CRNL \\
\hline 157 & $\mathrm{C} 068$ & $\mathrm{CP} 17$ & $31^{\circ} 37^{\prime} 59^{\prime \prime}$ & $90^{\circ} 31^{\prime} 12^{\prime \prime}$ & 23 & CRNL \\
\hline 158 & $\mathrm{C} 069$ & CP 66A & $31^{\circ} 37^{\prime} 35^{\prime \prime}$ & $90^{\circ} 31^{\prime} 05^{\prime \prime}$ & 30 & CRNL \\
\hline 159 & $\mathrm{C} 070$ & CP 30 & $31^{\circ} 38^{\prime} 31^{\prime \prime}$ & $90^{\circ} 31^{\prime} 35^{\prime \prime}$ & 67 & CRNL \\
\hline 160 & G385 & $\mathrm{CPC}$ & $31^{\circ} 35^{\prime} 32^{\prime \prime}$ & $90^{\circ} 29^{\prime} 29^{\prime \prime}$ & 65 & CRNL \\
\hline 161 & G386 & $\mathrm{CPC4}$ & $31^{\circ} 35^{\prime} 32^{\prime \prime}$ & $90^{\circ} 29^{\prime} 17^{\prime \prime}$ & 65 & CRNL \\
\hline 162 & G387 & $\mathrm{CPC}$ & $31^{\circ} 35^{\prime} 19^{\prime \prime}$ & $90^{\circ} 29^{\prime} 28^{\prime \prime}$ & 10 & CRNL \\
\hline 163 & G388 & $\mathrm{CPC5}$ & $31^{\circ} 35^{\prime} 11^{\prime \prime}$ & $90^{\circ} 29^{\prime} 24^{\prime \prime}$ & 73 & CRNL \\
\hline 164 & G389 & $\mathrm{CPG}$ & $31^{\circ} 35^{\prime} 42^{\prime \prime}$ & $90^{\circ} 31^{\prime} 53^{\prime \prime}$ & 45 & CRNL \\
\hline 165 & G390 & CP A & $31^{\circ} 35^{\prime} 36^{\prime \prime}$ & $90^{\circ} 30^{\prime} 53^{\prime \prime}$ & 52 & CRNL \\
\hline 166 & G391 & CP B2 & $31^{\circ} 35^{\prime} 30^{\prime \prime}$ & $90^{\circ} 30^{\prime} 44^{\prime \prime}$ & 36 & CRNL \\
\hline 167 & G392 & CP B3 & $31^{\circ} 35^{\prime} 19^{\prime \prime}$ & $90^{\circ} 30^{\prime} 28^{\prime \prime}$ & 50 & CRNL \\
\hline 168 & G393 & CP 5 & $31^{\circ} 34^{\prime} 51^{\prime \prime}$ & $90^{\circ} 30^{\prime} 28^{\prime \prime}$ & 40 & CRNL \\
\hline 169 & G394 & CPE & $31^{\circ} 34^{\prime} 44^{\prime \prime}$ & $90^{\circ} 30^{\prime} 30^{\prime \prime}$ & 60 & CRNL \\
\hline 170 & G395 & CP 41 & $31^{\circ} 34^{\prime} 29^{\prime \prime}$ & $90^{\circ} 30^{\prime} 42^{\prime \prime}$ & 65 & CRNL \\
\hline 171 & G396 & CP 42 & $31^{\circ} 34^{\prime} 17^{\prime \prime}$ & $90^{\circ} 30^{\prime} 41^{\prime \prime}$ & 35 & CRNL \\
\hline 172 & G397 & CP 7 & $31^{\circ} 34^{\prime} 46^{\prime \prime}$ & $90^{\circ} 31^{\prime} 04^{\prime \prime}$ & 57 & CRNL \\
\hline 173 & G398 & $\mathrm{CP} 8$ & $31^{\circ} 34^{\prime} 11^{\prime \prime}$ & $90^{\circ} 31^{\prime} 30^{\prime \prime}$ & 31 & CRNL \\
\hline 174 & G399 & CP 67 & $31^{\circ} 33^{\prime} 52^{\prime \prime}$ & $90^{\circ} 31^{\prime} 55^{\prime \prime}$ & 80 & CRNL \\
\hline 175 & G400 & CP 68B & $31^{\circ} 34^{\prime} 02^{\prime \prime}$ & $90^{\circ} 31^{\prime} 44^{\prime \prime}$ & 77 & CRNL \\
\hline 176 & G401 & CP 12 & $31^{\circ} 36^{\prime} 32^{\prime \prime}$ & $90^{\circ} 31^{\prime} 36^{\prime \prime}$ & 72 & CRNL \\
\hline 177 & G402 & $C P G$ & $31^{\circ} 33^{\prime} 09^{\prime \prime}$ & $90^{\circ} 31^{\prime} 53^{\prime \prime}$ & 75 & CRNL \\
\hline
\end{tabular}


penetrometer and a hydraulic press. The samples were analyzed at the USGS Water Resources Division Water-Quality Service Unit in Ocala, Florida.

Laboratory procedures used to analyze the water samples for this study were similar to the procedures used in the previous study by Kalkhoff (1986). The Water-Quality Service Unit used methods described by Fishman and Friedman (1989). The detection limits of these techniques are listed below.

\begin{tabular}{|c|c|c|c|}
\hline $\begin{array}{l}\text { WATSTORE } \\
\text { code }\end{array}$ & $\begin{array}{l}\text { Property or } \\
\text { constituent }\end{array}$ & $\begin{array}{l}\text { Method } \\
\text { number }\end{array}$ & $\begin{array}{l}\text { Detection } \\
\text { limit }\end{array}$ \\
\hline 90095 & Specific conductance (lab) & $\mathrm{I}-1780-84$ & $1.0 \mu \mathrm{S} / \mathrm{cm}$ \\
\hline 00403 & $\mathrm{pH}$ (lab) & I-1586-84 & 0.1 standard $\mathrm{pH}$ unit \\
\hline 00930 & Sodium, dissolved & $\mathrm{I}-1735-85$ & $0.1 \mathrm{mg} / \mathrm{L}$ \\
\hline 00940 & Chloride, dissolved & I-2057-84 & $0.1 \mathrm{mg} / \mathrm{L}$ \\
\hline 01005 & Barium, dissolved & $\mathrm{I}-1472-87$ & $5.0 \mu \mathrm{g} / \mathrm{L}$ \\
\hline 01080 & Strontium, dissolved & $\mathrm{I}-1472-87$ & $2.0 \mu \mathrm{g} / \mathrm{L}$ \\
\hline
\end{tabular}

All the water-quality data are stored in WATSTORE, the National Water Data Storage and Retrieval System of the U.S. Geological Survey. The system resides on the central computer facilities of the USGS at its National Center in Restori, Virginia.

\section{CHEMICAL QUALITY OF BRINE AND UNCONTAMINATED GROUND WATER}

Analyses of three brine samples from the major oil-producing formation (the lower part of the Tuscaloosa Formation) in the Brookhaven oil field are summarized in table 2 . The brine samples represent water between a depth of 10,340 to $10,510 \mathrm{ft}$. Sodium is the predominant cation and chloride the predominant anion in the water. Sodium concentrations ranged from 46,700 to $48,700 \mathrm{mg} / \mathrm{L}$ with a mean concentration of $48,000 \mathrm{mg} / \mathrm{L}$. Chloride concentrations ranged from 95,900 to $100,100 \mathrm{mg} / \mathrm{L}$ with a mean concentration of $98,500 \mathrm{mg} / \mathrm{L}$. The mean dissolved-solids concentration of the brine was about $160,300 \mathrm{mg} / \mathrm{L}$. The sodium to chloride ratio in the brine was about 0.49 . The brine contains relatively large concentrations of barium plus strontium (mean of $1,220 \mathrm{mg} / \mathrm{L}$ ), constituents that normally occur in very small concentrations in shallow and moderately deep ground water and in streams.

Sodium is the predominant cation and chloride and bicarbonate are the predominant anions in uncontaminated water in the Citronelle aquifer in northwestern Lincoln County (Kalkhoff, 1986). Sodium concentrations ranged from 2.7 to $14 \mathrm{mg} / \mathrm{L}$ (median, $4.5 \mathrm{mg} / \mathrm{L}$ ) for 10 samples collected during August-September 1984 (table 3). Chloride concentrations ranged from 3.4 to $21 \mathrm{mg} / \mathrm{L}$ (median, $5.9 \mathrm{mg} / \mathrm{L}$ ). The sodium to chloride ratio ranged 
Table 2. Summary of analyses of brine samples from the Brookhaven oil field, Lincoln County, Mississippi

[From Hawkins and others, 1963. Dissolved solids, stoichiometrical analysis; mg/L, milligrams per liter. All constituents dissolved]

Lower part of the Tuscaloosa Formation

(3 samples)

Depth, in feet

Dissolved solids (mg/L)

Calcium (mg/L)

Magnesium (mg/L)

Sodium (mg/L)

Bicarbonate (mg/L)

Sulfate $(\mathrm{mg} / \mathrm{L})$

Chloride (mg/L)

Barium and strontium (mg/L)

Sodium to chloride ratio

$\begin{array}{ccc}\text { Minimum } & \text { Mean } & \text { Maximum } \\ 10,340 & 10,420 & 10,510 \\ 156,700 & 160,300 & 162,600 \\ 12,100 & 12,600 & 12,900 \\ 780 & 990 & 1,100 \\ 46,700 & 48,000 & 48,700 \\ 0 & 26 & 77 \\ 0 & 98 & 160 \\ 95,900 & 98,500 & 100,100 \\ 34 & 1,220 & 2,073 \\ 0.49 & 0.49 & 0.49\end{array}$

Table 3. Summary of water-quality data for uncontaminated ground water in the Citronelle aquifer in northwestern Lincoln County, Mississippi

[From Kalkhoff, 1986; $\mu \mathrm{S} / \mathrm{cm}$, microsiemens per centimeter at 25 degrees Celsius; mg/L, milligrams per liter; $\mu \mathrm{g} / \mathrm{L}$, micrograms per liter. All constituents dissolved]

Depth, in feet

Specific conductance, $(\mu \mathrm{S} / \mathrm{cm})$

$\mathrm{pH}$

Sodium (mg/L)

Chloride ( $\mathrm{mg} / \mathrm{L})$

Barium $(\mu \mathrm{g} / \mathrm{L})$

Strontium $(\mu \mathrm{g} / \mathrm{L})$

Sodium to chloride ratio

\section{(10 samples)}

$\begin{array}{ccc}\text { Minimum } & \text { Median } & \text { Maximum } \\ 40 & 80 & 120 \\ 25 & 44 & 86 \\ 4.9 & 5.2 & 5.4 \\ 2.7 & 4.5 & 14 \\ 3.4 & 5.9 & 21 \\ 20 & 31 & 50 \\ 6 & 10 & 20 \\ 0.57 & 0.80 & 1.03\end{array}$


from 0.57 to $1.03 \mathrm{mg} / \mathrm{L}$ (median, 0.80 ). A chloride concentration of $20 \mathrm{mg} / \mathrm{L}$ or less is considered background level for water from the Citronelle aquifer for purposes of this study. Kalkhoff concluded that, for the study area, water with a chloride concentration exceeding $20 \mathrm{mg} / \mathrm{L}$ and a sodium to chloride ratio of less than 0.60 probably is contaminated. Additional indicators of probable brine contamination, according to Kalkhoff, are a barium concentration greater than $50 \mu \mathrm{g} / \mathrm{L}$ and a strontium concentration greater than $20 \mu \mathrm{g} / \mathrm{L}$.

Uncontaminated water in the Miocene aquifer system in the study area is quite similar to uncontaminated water in the Citronelle aquifer. Consequently, Kalkhoff (1986) concluded that, for the study area, indicators of probable brine contamination are the same as those for the Citronelle aquifer.

\section{BRINE CONTAMINATION OF GROUND WATER}

Disposal of brine by injection into formations containing water with dissolved-solids concentrations greater than $10,000 \mathrm{mg} / \mathrm{L}$ can adversely affect shallow ground-water quality, even though this practice is environmentally more acceptable than surface disposal. Brine may leak directly into shallow aquifers or move upward through the annular space outside the well casing of improperly constructed and maintained or deteriorated injection wells. Brine may also leak through the deteriorated casing of deep abandoned wells into shallow freshwater aquifers.

\section{Brine Contamination during August 1993-December 1994}

Water-quality data for the selected 177 ground-water samples collected during this study (August 1993-December 1994) from wells in northwestern Lincoln County are listed in table 4 and summarized in table 5 . Sodium concentrations ranged from 2.2 to $3,700 \mathrm{mg} / \mathrm{L}$ (median, $14 \mathrm{mg} / \mathrm{L}$ ). Chloride concentrations ranged from 2.5 to $9,400 \mathrm{mg} / \mathrm{L}$ (median, $21 \mathrm{mg} / \mathrm{L}$ ). Water from 89 wells ( 50 percent) was considered contaminated by brine--that is, had chloride concentrations greater than $20 \mathrm{mg} / \mathrm{L}$.

The chloride concentrations in water from only 12 wells (7 percent) sampled during this study were greater than $250 \mathrm{mg} / \mathrm{L}$, which is the recommended secondary limit for drinking water (U.S. Environmental Protection Agency, 1986a, b). [The recommended limit for chloride in public water supplies is based largely on aesthetic reasons.]

Water-quality data in the following sections are discussed on the basis of depth below land surface rather than on the basis of aquifer. The primary reason for this is that, although there is some uncertainty from which aquifer the water samples originated for a few wells, water-level data indicate that the Citronelle and Miocene aquifers may be hydraulically connected in part of the study area (Kalkhoff, 1986).

The areal extent of brine contamination (chloride concentration equal to or greater than $20 \mathrm{mg} / \mathrm{L}$ ) in shallow ground water (depth $120 \mathrm{ft}$ or less) in the vicinity of the Brookhaven oil field during August 1993-December 1994 is 
Table 4. Water-quality data for selected wells in Lincoln County, Mississippi (August 1993-December 1994)

[ft, feet; $\mu \mathrm{S} / \mathrm{cm}$, microsiemens per centimeter at 25 degrees Celsius; $\mathrm{mg} / \mathrm{L}$, milligrams per liter; $\mu \mathrm{g} / \mathrm{L}$. micrograms per liter. All constituents dissolved]

\begin{tabular}{|c|c|c|c|c|c|c|c|c|c|}
\hline $\begin{array}{l}\text { Site } \\
\text { number }\end{array}$ & $\begin{array}{c}\text { Local } \\
\text { number }\end{array}$ & Date & $\begin{array}{c}\text { Depth } \\
(\mathrm{ft})\end{array}$ & $\begin{array}{c}\text { Specific } \\
\text { conductance } \\
\text { (lab, } \\
\mu \mathrm{S} / \mathrm{cm})\end{array}$ & $\begin{array}{c}\mathrm{pH} \\
\text { (abo, } \\
\text { standard } \\
\text { units) }\end{array}$ & $\begin{array}{l}\text { Sodium } \\
\text { (mg/L) }\end{array}$ & $\begin{array}{l}\text { Chloride } \\
\text { (mg/L) }\end{array}$ & $\begin{array}{l}\text { Barium } \\
(\mu \mathrm{g} / \mathrm{L})\end{array}$ & $\begin{array}{c}\text { Strontium } \\
(\mu \mathrm{g} / \mathrm{L})\end{array}$ \\
\hline 1 & B37 & 090293 & 152 & 40 & 5.7 & 4.7 & 5.1 & 27 & 5 \\
\hline 2 & B48 & 012694 & 80 & 61 & 5.5 & 6.8 & 10 & 38 & 14 \\
\hline 3 & $\mathrm{C} 17$ & 011894 & 50 & 134 & 5.6 & 15 & 40 & 69 & 18 \\
\hline 4 & C18 & 011894 & 65 & 160 & 6.0 & 18 & 34 & 110 & 23 \\
\hline 5 & C19 & 090893 & 63 & 720 & 6.0 & 81 & 200 & 260 & 95 \\
\hline 6 & $\mathrm{C} 20$ & 011894 & 100 & 43 & 5.6 & 4.8 & 8.3 & 34 & 8 \\
\hline 7 & $\mathrm{C} 21$ & 020394 & 40 & 396 & 6.8 & 52 & 30 & 99 & 88 \\
\hline 8 & $\mathrm{C} 22$ & 011994 & 200 & 116 & 5.3 & 15 & 18 & 81 & 35 \\
\hline 9 & $\mathrm{C} 24$ & 011994 & 49 & 106 & 5.8 & 18 & 16 & 21 & 6 \\
\hline 10 & $\mathrm{C} 25$ & 011994 & 60 & 124 & 5.3 & 14 & 29 & 46 & 19 \\
\hline 11 & $\mathrm{C} 28$ & 011894 & 200 & 77 & 6.5 & 5.5 & 3.0 & 60 & 63 \\
\hline 12 & $\mathrm{C} 29$ & 011294 & 80 & 352 & 5.7 & 25 & 99 & 450 & 120 \\
\hline 13 & C31 & 012094 & 60 & 412 & 5.8 & 40 & 110 & 260 & 75 \\
\hline 14 & C32 & 012094 & 100 & 55 & 5.4 & 7.1 & 7.5 & 21 & 8 \\
\hline 15 & C34 & 011994 & 100 & 62 & 5.9 & 8.2 & 8.4 & 19 & 7 \\
\hline 16 & C35 & 090893 & 70 & 476 & 5.4 & 54 & 140 & 410 & 74 \\
\hline 17 & C 36 & 012094 & 30 & 161 & 5.0 & 12 & 21 & 170 & 83 \\
\hline 18 & C37 & 012194 & 75 & 420 & 5.3 & 39 & 120 & 400 & 85 \\
\hline 19 & C45 & 011894 & 28 & 66 & 5.8 & 3.6 & 3.9 & 64 & 42 \\
\hline 20 & C49 & 090893 & 87 & 55 & 5.6 & 5.9 & 11 & 52 & 5 \\
\hline 21 & C51 & 012194 & 115 & 29 & 6.1 & 3.4 & 3.0 & 21 & 7 \\
\hline 22 & C52 & 011294 & 61 & 95 & 5.6 & 14 & 21 & 42 & 8 \\
\hline 23 & C53 & 020294 & 90 & 48 & 5.5 & 6.0 & 8.9 & 26 & 9 \\
\hline 24 & C55 & 011194 & 87 & 80 & 5.6 & 8.8 & 18 & 62 & 12 \\
\hline 25 & C58 & 020394 & 64 & 103 & 5.8 & 16 & 19 & 35 & 10 \\
\hline 26 & C61 & 011194 & 89 & 37 & 5.8 & 3.7 & 4.8 & 28 & 11 \\
\hline 27 & C62 & 011194 & 60 & 66 & 6.2 & 9.2 & 11 & 17 & 8 \\
\hline 28 & F20 & 012594 & 85 & 39 & 5.6 & 3.8 & 5.7 & 32 & 10 \\
\hline 29 & F21 & 012694 & 80 & 88 & 5.6 & 10 & 18 & 59 & 19 \\
\hline 30 & F24 & 012594 & 87 & 43 & 5.8 & 4.4 & 6.2 & 24 & 10 \\
\hline 31 & F27 & 012594 & 98 & 49 & 5.5 & 6.0 & 8.2 & 26 & 7 \\
\hline 32 & F28 & 012594 & 60 & 55 & 5.6 & 7.7 & 9.3 & 23 & 7 \\
\hline 33 & F38 & 012694 & 72 & 38 & 5.6 & 5.1 & 6.7 & 20 & 6 \\
\hline 34 & F39 & 012594 & 80 & 27 & 5.8 & 3.1 & 3.4 & 20 & 8 \\
\hline 35 & F40 & 012794 & 120 & 51 & 5.8 & 7.0 & 7.1 & 28 & 12 \\
\hline
\end{tabular}


Table 4. Water-quality data for selected wells in Lincoln County, Mississippi (August 1993-December 1994)--Continued

\begin{tabular}{|c|c|c|c|c|c|c|c|c|c|}
\hline $\begin{array}{l}\text { Site } \\
\text { number }\end{array}$ & $\begin{array}{l}\text { Local } \\
\text { number }\end{array}$ & Date & $\begin{array}{c}\text { Depth } \\
\text { (ft) }\end{array}$ & $\begin{array}{c}\text { Specific } \\
\text { conductance } \\
\text { (lab, } \\
\mu \mathrm{S} / \mathrm{cm})\end{array}$ & $\begin{array}{c}\mathrm{pH} \\
\text { alab, } \\
\text { standard } \\
\text { units) }\end{array}$ & $\begin{array}{l}\text { Sodium } \\
\text { (mg/L) }\end{array}$ & $\begin{array}{l}\text { Chloride } \\
\text { (mg/L) }\end{array}$ & $\begin{array}{l}\text { Barium } \\
(\mu \mathrm{g} / \mathrm{L})\end{array}$ & $\begin{array}{l}\text { Strontium } \\
(\mu \mathrm{g} / \mathrm{L})\end{array}$ \\
\hline 36 & F41 & 012694 & 80 & 28 & 5.7 & 2.2 & 3.6 & 27 & 10 \\
\hline 37 & F44 & 013194 & 70 & 128 & 5.4 & 15 & 31 & 77 & 25 \\
\hline 38 & F48 & 020494 & 67 & 33 & 5.5 & 3.3 & 4.1 & 19 & 5 \\
\hline 39 & G25 & 082093 & 150 & 66 & 6.1 & 7.1 & 5.5 & 42 & 25 \\
\hline 40 & G57 & 082793 & 91 & 60 & 5.8 & 8.0 & 11 & 39 & $<1$ \\
\hline 41 & G58 & 013194 & 80 & 326 & 6.1 & 38 & 80 & 110 & 51 \\
\hline 42 & G59 & 012694 & 76 & 854 & 5.7 & 110 & 240 & 410 & 140 \\
\hline 43 & G60 & 122993 & 100 & 1,260 & 5.5 & 160 & 400 & 710 & 230 \\
\hline 44 & G61 & 122993 & 85 & 649 & 5.5 & 59 & 190 & 440 & 170 \\
\hline 45 & G62 & 010494 & 45 & 7,200 & 6.1 & 1,200 & 2,500 & 2,100 & 7,500 \\
\hline 46 & G63 & 012694 & 65 & 64 & 5.5 & 9.0 & 11 & 43 & 11 \\
\hline 47 & G66 & 020494 & 48 & 141 & 5.5 & 21 & 33 & 18 & 68 \\
\hline 48 & G67 & 082593 & 150 & 69 & 6.2 & 7.0 & 10 & 60 & 33 \\
\hline 49 & G68 & 122193 & 308 & 231 & 6.0 & 25 & 58 & 210 & 120 \\
\hline 50 & G69 & 020294 & 85 & 272 & 5.3 & 32 & 74 & 160 & 42 \\
\hline 51 & G71 & 082693 & 256 & 85 & 6.4 & 10 & 3.4 & 53 & 56 \\
\hline 52 & $\mathrm{G} 72$ & 082093 & 80 & 54 & 6.1 & 5.9 & 8.3 & 41 & $<1$ \\
\hline 53 & G73 & 081993 & 120 & 50 & 6.0 & 6.2 & 5.3 & 38 & $<1$ \\
\hline 54 & G74 & 082693 & 120 & 43 & 6.1 & 4.0 & 3.5 & 38 & 5 \\
\hline 55 & G75 & 082693 & 100 & 42 & 6.1 & 3.9 & 3.5 & 29 & 16 \\
\hline 56 & G76 & 081993 & 100 & 52 & 6.0 & 7.3 & 5.2 & 32 & $<1$ \\
\hline 57 & G77 & 082093 & 100 & 161 & 5.5 & 21 & 41 & 65 & 10 \\
\hline 58 & G79 & 082093 & 75 & 98 & 6.2 & 8.8 & 12 & 43 & 20 \\
\hline 59 & G80 & 081993 & 118 & 121 & 5.8 & 14 & 28 & 78 & 18 \\
\hline 60 & G82 & 081993 & 45 & 104 & 5.4 & 13 & 25 & 45 & 4 \\
\hline 61 & G83 & 010494 & 50 & 316 & 5.4 & 41 & 94 & 130 & 60 \\
\hline 62 & G85 & 082693 & 100 & 257 & 5.8 & 37 & 55 & 97 & 16 \\
\hline 63 & G86 & 082693 & 65 & 256 & 5.9 & 39 & 59 & 83 & 22 \\
\hline 64 & G87 & 020294 & 60 & 35 & 5.5 & 4.2 & 4.5 & 28 & 7 \\
\hline 65 & G88 & 082793 & 80 & 36 & 5.5 & 4.8 & 6.6 & 19 & $<1$ \\
\hline 66 & G89 & 083193 & 50 & 196 & 5.9 & 13 & 32 & 130 & 72 \\
\hline 67 & G91 & 013194 & 106 & 37 & 5.7 & 5.0 & 5.9 & 25 & 5 \\
\hline 68 & G95 & 020194 & 30 & 37 & 5.6 & 5.1 & 5.8 & 23 & 7 \\
\hline 69 & G97 & 020194 & 175 & 54 & 5.9 & 5.4 & 6.4 & 65 & 19 \\
\hline 70 & G99 & 020394 & 48 & 994 & 5.9 & 110 & 280 & 1,100 & 370 \\
\hline
\end{tabular}


Table 4. Water-quality data for selected wells in Lincoln County, Mississipfi (August 1993-December 1994)--Continued

\begin{tabular}{|c|c|c|c|c|c|c|c|c|c|}
\hline $\begin{array}{l}\text { Site } \\
\text { number }\end{array}$ & $\begin{array}{l}\text { Local } \\
\text { number }\end{array}$ & Date & $\begin{array}{c}\text { Depth } \\
(\mathrm{ft})\end{array}$ & $\begin{array}{c}\text { Specific } \\
\text { conductance } \\
\text { (lab, } \\
\mu \mathrm{S} / \mathrm{cm})\end{array}$ & $\begin{array}{c}\mathrm{pH} \\
\text { (lab, } \\
\text { standard } \\
\text { units) }\end{array}$ & $\begin{array}{l}\text { Sodium } \\
\text { (mg/L) }\end{array}$ & $\begin{array}{l}\text { Chloride } \\
\text { (mg/L) }\end{array}$ & $\begin{array}{l}\text { Barium } \\
(\mu \mathrm{g} / \mathrm{L})\end{array}$ & $\begin{array}{l}\text { Strontium } \\
(\mu \mathrm{g} / \mathrm{L})\end{array}$ \\
\hline 71 & G100 & 020394 & 90 & 276 & 5.5 & 37 & 74 & 150 & 40 \\
\hline 72 & G106 & 020394 & 92 & 271 & 6.7 & 33 & 50 & 44 & 40 \\
\hline 73 & G107 & 122193 & 120 & 720 & 5.6 & 110 & 210 & 420 & 160 \\
\hline 74 & G109 & 020194 & 79 & 292 & 5.9 & 38 & 66 & 82 & 33 \\
\hline 75 & G110 & 020194 & 100 & 97 & 5.8 & 10 & 18 & 73 & 21 \\
\hline 76 & G114 & 122993 & 84 & 1,900 & 5.4 & 260 & 590 & 1,100 & 530 \\
\hline 77 & G117 & 013194 & 73 & 106 & 5.4 & 13 & 26 & 72 & 21 \\
\hline 78 & G118 & 020394 & 69 & 187 & 6.0 & 27 & 39 & 43 & 51 \\
\hline 79 & G119 & 090893 & 98 & 134 & 5.9 & 17 & 32 & 73 & 15 \\
\hline 80 & G120 & 090893 & 85 & 256 & 5.9 & 36 & 57 & 61 & 21 \\
\hline 81 & G123 & 090193 & 75 & 365 & 5.3 & 56 & 100 & 140 & 56 \\
\hline 82 & G127 & 013194 & 70 & 642 & 5.4 & 82 & 180 & 320 & 110 \\
\hline 83 & G131 & 020394 & 200 & 2,750 & 5.2 & 280 & 860 & 2,800 & 1,200 \\
\hline 84 & G133 & 082593 & 120 & 50 & 6.2 & 5.3 & 5.1 & 44 & 10 \\
\hline 85 & G134 & 122193 & 50 & 74 & 5.8 & 13 & 14 & 23 & 6 \\
\hline 86 & G135 & 082593 & 140 & 46 & 6.2 & 5.0 & 5.0 & 32 & 12 \\
\hline 87 & G137 & 082593 & 75 & 354 & 5.6 & 42 & 96 & 150 & 56 \\
\hline 88 & G138 & 082693 & 85 & 367 & 5.5 & 40 & 99 & 190 & 65 \\
\hline 89 & G139 & 122993 & 93 & 291 & 5.5 & 37 & 87 & 130 & 45 \\
\hline 90 & G140 & 090193 & 80 & 437 & 5.2 & 52 & 120 & 250 & 140 \\
\hline 91 & G142 & 090193 & 40 & 24 & 5.6 & 2.9 & 3.4 & 17 & $<1$ \\
\hline 92 & G143 & 122193 & 80 & 100 & 6.2 & 8.1 & 14 & 44 & 42 \\
\hline 93 & G144 & 090193 & 75 & 58 & 5.8 & 6.6 & 6.2 & 23 & 8 \\
\hline 94 & G148 & 021794 & 68 & 40 & 5.6 & 5.4 & 6.7 & 23 & 9 \\
\hline 95 & G151 & 022894 & 180 & 36 & 5.9 & 4.0 & 3.3 & 26 & 14 \\
\hline 96 & G152 & 022894 & 165 & 475 & 5.1 & 54 & 140 & 330 & 130 \\
\hline 97 & G161 & 030194 & 62 & 56 & 5.5 & 7.8 & 10 & 29 & 9 \\
\hline 98 & G162 & 030294 & 128 & 56 & 6.0 & 5.2 & 6.3 & 68 & 24 \\
\hline 99 & G163 & 022894 & 87 & 188 & 5.6 & 19 & 50 & 230 & 57 \\
\hline 100 & G165 & 030194 & 114 & 698 & 5.5 & 67 & 210 & 660 & 260 \\
\hline 101 & G169 & 022894 & 115 & 137 & 5.8 & 15 & 31 & 86 & 19 \\
\hline 102 & G175 & 083193 & 70 & 90 & 5.4 & 12 & 20 & 36 & 11 \\
\hline 103 & G177 & 082593 & 120 & 28 & 5.8 & 2.6 & 2.6 & 31 & $<1$ \\
\hline 104 & G178 & 082793 & 60 & 32 & 5.6 & 3.7 & 4.2 & 34 & $<1$ \\
\hline 105 & G180 & 082493 & 147 & 327 & 5.8 & 36 & 89 & 260 & 76 \\
\hline
\end{tabular}


Table 4. Water-quality data for selected wells in Lincoln County, Mississippi (August 1993-December 1994)--Continued

\begin{tabular}{|c|c|c|c|c|c|c|c|c|c|}
\hline $\begin{array}{l}\text { Site } \\
\text { number }\end{array}$ & $\begin{array}{l}\text { Local } \\
\text { number }\end{array}$ & Date & $\begin{array}{l}\text { Depth } \\
(\mathrm{ft})\end{array}$ & $\begin{array}{c}\text { Specific } \\
\text { conductance } \\
\text { (ab, } \\
\mu \mathrm{S} / \mathrm{cm})\end{array}$ & $\begin{array}{c}\text { pH } \\
\text { (lab, } \\
\text { standard } \\
\text { units) }\end{array}$ & $\begin{array}{l}\text { Sodium } \\
\text { (mg/L) }\end{array}$ & $\begin{array}{l}\text { Chloride } \\
\text { (mg/L) }\end{array}$ & $\begin{array}{l}\text { Barium } \\
(\mu \mathrm{g} / \mathrm{L})\end{array}$ & $\begin{array}{l}\text { Strontium } \\
(\mu \mathrm{g} / \mathrm{L})\end{array}$ \\
\hline 106 & G181 & 122993 & 150 & 267 & 5.7 & 31 & 78 & 220 & 140 \\
\hline 107 & G182 & 082493 & 148 & 31 & 5.9 & 3.3 & 5.3 & 40 & 5 \\
\hline 108 & G186 & 090193 & 150 & 45 & 6.2 & 4.7 & 4.5 & 100 & 16 \\
\hline 109 & G208 & 083093 & 160 & 2,500 & 5.2 & 320 & 800 & 2,500 & 1,200 \\
\hline 110 & G209 & 083093 & 120 & 1,530 & 4.9 & 380 & 460 & 400 & 1,400 \\
\hline 111 & G210 & 083093 & 65 & 4,860 & 5.7 & 700 & 1,600 & 3,300 & 2,500 \\
\hline 112 & G211 & 083093 & 125 & 168 & 5.8 & 15 & 41 & 190 & 81 \\
\hline 113 & G212 & 083193 & 120 & 301 & 5.4 & 34 & 82 & 230 & 55 \\
\hline 114 & G213 & 090193 & 80 & 365 & 6.0 & 44 & 95 & 110 & 96 \\
\hline 115 & G214 & 090193 & 65 & 963 & 5.8 & 130 & 290 & 400 & 400 \\
\hline 116 & G215 & 090193 & 80 & 127 & 5.9 & 18 & 25 & 42 & 8 \\
\hline 117 & G216 & 090193 & 68 & 432 & 6.1 & 67 & 120 & 120 & 33 \\
\hline 118 & G217 & 090293 & 60 & 744 & 5.1 & 94 & 220 & 470 & 200 \\
\hline 119 & G218 & 090293 & 70 & 352 & 5.4 & 51 & 99 & 200 & 49 \\
\hline 120 & G247 & 021794 & 160 & 133 & 6.0 & 22 & 24 & 27 & 10 \\
\hline 121 & G258 & 021894 & 145 & 46 & 6.1 & 3.8 & 3.7 & 75 & 17 \\
\hline 122 & G262 & 021494 & 160 & 51 & 6.1 & 4.7 & 4.4 & 54 & 25 \\
\hline 123 & G267 & 021494 & 113 & 46 & 6.1 & 4.6 & 3.7 & 61 & 18 \\
\hline 124 & G268 & 021594 & 126 & 70 & 5.9 & 6.9 & 12 & 74 & 31 \\
\hline 125 & G271 & 021594 & 66 & 99 & 5.7 & 12 & 22 & 49 & 14 \\
\hline 126 & G272 & 021494 & 58 & 98 & 5.5 & 12 & 21 & 62 & 19 \\
\hline 127 & G275 & 021594 & 84 & 109 & 5.8 & 14 & 24 & 61 & 15 \\
\hline 128 & G276 & 021594 & 90 & 113 & 6.0 & 16 & 22 & 31 & 19 \\
\hline 129 & G279 & 021694 & 85 & 38 & 5.8 & 3.8 & 4.6 & 33 & 8 \\
\hline 130 & G283 & 021594 & 78 & 27 & 5.6 & 2.5 & 2.5 & 36 & 12 \\
\hline 131 & G312 & 021694 & 93 & 156 & 5.6 & 19 & 39 & 59 & 21 \\
\hline 132 & G316 & 021794 & 160 & 46 & 5.8 & 5.7 & 6.4 & 32 & 9 \\
\hline 133 & G324 & 021594 & 120 & 47 & 5.7 & 5.0 & 5.2 & 36 & 24 \\
\hline 134 & G333 & 021694 & 160 & 45 & 5.9 & 4.9 & 5.1 & 44 & 18 \\
\hline 135 & G335 & 021694 & 180 & 51 & 6.1 & 5.7 & 4.9 & 57 & 18 \\
\hline 136 & G337 & 021494 & 170 & 717 & 5.9 & 69 & 200 & 380 & 150 \\
\hline 137 & G346 & 021694 & 170 & 31 & 5.8 & 3.0 & 2.6 & 31 & 14 \\
\hline 138 & G354 & 021794 & 89 & 58 & 5.7 & 9.1 & 11 & 32 & 8 \\
\hline 139 & G356 & 021894 & 107 & 134 & 5.6 & 16 & 33 & 81 & 22 \\
\hline 140 & G365 & 021794 & 86 & 366 & 5.1 & 45 & 100 & 260 & 100 \\
\hline
\end{tabular}


Table 4. Water-quality data for selected wells in Lincoln County, Mississippi (August 1993-December 1994)--Continued

\begin{tabular}{|c|c|c|c|c|c|c|c|c|c|}
\hline $\begin{array}{l}\text { Site } \\
\text { number }\end{array}$ & $\begin{array}{l}\text { Local } \\
\text { number }\end{array}$ & Date & $\begin{array}{c}\text { Depth } \\
(\mathrm{ft})\end{array}$ & $\begin{array}{c}\text { Specific } \\
\text { conductance } \\
(\text { lab, } \\
\mu \mathrm{S} / \mathrm{cm})\end{array}$ & $\begin{array}{c}\text { pH } \\
\text { (lab, } \\
\text { standard } \\
\text { units) }\end{array}$ & $\begin{array}{l}\text { Sodium } \\
\text { (mg/L) }\end{array}$ & $\begin{array}{l}\text { Chloride } \\
\text { (mg/L) }\end{array}$ & $\begin{array}{l}\text { Barium } \\
(\mu \mathrm{g} / \mathrm{L})\end{array}$ & $\begin{array}{c}\text { Strontium } \\
(\mu \mathrm{g} / \mathrm{L})\end{array}$ \\
\hline 141 & G368 & 030294 & 85 & 148 & 5.5 & 23 & 37 & 42 & 10 \\
\hline 142 & G370 & 021894 & 86 & 329 & 5.4 & 46 & 94 & 170 & 38 \\
\hline 143 & G377 & 021494 & 160 & 46 & 6.0 & 5.0 & 5.1 & 37 & 20 \\
\hline 144 & G379 & 011294 & 160 & 29 & 5.9 & 2.9 & 2.7 & 29 & 10 \\
\hline 145 & G380 & 020494 & 165 & 85 & 6.3 & 14 & 3.1 & 32 & 28 \\
\hline 146 & G381 & 030294 & 93 & 89 & 5.7 & 13 & 19 & 28 & 9 \\
\hline 147 & G382 & 030294 & 58 & 127 & 5.8 & 14 & 27 & 46 & 66 \\
\hline 148 & G383 & 030294 & 57 & 73 & 5.6 & 8.2 & 12 & 26 & 16 \\
\hline 149 & G403 & 120894 & 110 & 51 & 6.1 & 5.1 & 7.2 & -. & -. \\
\hline 150 & G404 & 120894 & 40 & 99 & 6.2 & 16 & 18 & -- & -- \\
\hline 151 & K35 & 010494 & 80 & 97 & 6.0 & 15 & 13 & 34 & 19 \\
\hline 152 & C63 & 101394 & 30 & 115 & 6.2 & 20 & 24 & -- & -- \\
\hline 153 & C64 & 102794 & 16 & 95 & 6.3 & 12 & 12 & -. & -. \\
\hline 154 & C65 & 101294 & 57 & 131 & 6.4 & 20 & 23 & -. & -- \\
\hline 155 & C66 & 102594 & 26 & 112 & 6.2 & 16 & 19 & -. & -. \\
\hline 156 & C67 & 102494 & 45 & 138 & 6.4 & 22 & 24 & -. & -. \\
\hline 157 & C68 & 102094 & 23 & 178 & 6.1 & 22 & 29 & -. & -- \\
\hline 158 & C69 & 102094 & 30 & 207 & 6.4 & 23 & 42 & -- & -- \\
\hline 159 & $\mathrm{C} 70$ & 110794 & 67 & 158 & 6.4 & 21 & 27 & -. & -. \\
\hline 160 & G385 & 101994 & 65 & 116 & 7.0 & 9.5 & 8.7 & -- & -- \\
\hline 161 & G386 & 101994 & 65 & 101 & 6.8 & 8.7 & 11 & -- & -- \\
\hline 162 & G387 & 101894 & 10 & 217 & 7.2 & 14 & 7.8 & -. & -- \\
\hline 163 & G388 & 101894 & 73 & 67 & 6.6 & 6.1 & 6.6 & -. & -. \\
\hline 164 & G389 & 110394 & 45 & 822 & 6.2 & 160 & 230 & -. & -- \\
\hline 165 & G390 & 110394 & 52 & 154 & 6.5 & 28 & 30 & -- & -- \\
\hline 166 & G391 & 102694 & 36 & 1,360 & 6.4 & 190 & 400 & -- & -- \\
\hline 167 & G392 & 102694 & 50 & 79 & 6.4 & 9.6 & 9.6 & -- & -. \\
\hline 168 & G393 & 103194 & 40 & 476 & 6.1 & 64 & 63 & -- & -- \\
\hline 169 & G394 & 110294 & 60 & 25,000 & 5.1 & 3,700 & 9,400 & -. & -- \\
\hline 170 & G395 & 110294 & 65 & 433 & 6.1 & 49 & 120 & -. & -- \\
\hline 171 & G396 & 110294 & 35 & 53 & 6.8 & 4.3 & 6.6 & -. & -- \\
\hline 172 & G397 & 110194 & 57 & 162 & 6.1 & 18 & 38 & -- & $\cdots$ \\
\hline 173 & G398 & 110194 & 31 & 75 & 6.2 & 11 & 12 & -- & -. \\
\hline 174 & G399 & 101794 & 80 & 118 & 6.5 & 17 & 23 & -. & -- \\
\hline 175 & G400 & 110894 & 77 & 1,120 & 6.1 & 140 & 330 & -- & - \\
\hline 176 & G401 & 110894 & 72 & 66 & 6.1 & 8.7 & 11 & -- & - \\
\hline 177 & G402 & 110994 & 75 & 122 & 6.5 & 21 & 24 & -- & -- \\
\hline
\end{tabular}


Table 5. Summary of water-quality data for selected wells in Lincoln County, Mississippi (August 1993-December 1994) $[\mu \mathrm{S} / \mathrm{cm}$, microsiemens per centimeter at 25 degrees Celsius; mg/L, milligrams per liter; $\mu \mathrm{g} / \mathrm{L}$, micrograms per liter. All constituents dissolved]

Depth, in feet

Specific conductance, lab $(\mu S / \mathrm{cm})$ $\mathrm{pH}$, lab

Sodium (mg/L)

Chloride (mg/L)

Barium $(\mu \mathrm{g} / \mathrm{L})$

Strontium $(\mu \mathrm{g} / \mathrm{L})$

Depth, in feet

Specific conductance, lab $(\mu \mathrm{S} / \mathrm{cm})$

$\mathrm{pH}$, lab

Sodium (mg/L)

Chloride (mg/L)

Barium $(\mu \mathrm{g} / \mathrm{L})$

Strontium ( $\mu \mathrm{g} / \mathrm{L})$
Shallow wells

(146 samples)

Minimum
10
24
4.9
2.2
2.5
17
$<1$

Deep wells

(31 samples)

Minimum
125
29
5.1
2.9
2.6
26
5

All wells

(177samples)

$\begin{array}{ccc}\text { Minimum } & \text { Median } & \text { Maximum } \\ 10 & 80 & 308 \\ 24 & 106 & 25,000 \\ 4.9 & 5.8 & 7.2 \\ 2.2 & 14 & 3,700 \\ 2.5 & 21 & 9,400 \\ 17 & 57 & 3,300 \\ <1 & 19 & 7,500\end{array}$

$\begin{array}{cc}\text { Median } & \text { Maximum } \\ 75 & 120 \\ 117 & 25,000 \\ 5.8 & 7.2 \\ 15 & 3,700 \\ 24 & 9,400 \\ 50 & 3,300 \\ 19 & 7,500\end{array}$

$\begin{array}{cc}\text { Median } & \text { Maximum } \\ 160 & 308 \\ 66 & 2,750 \\ 5.9 & 6.5 \\ 5.7 & 320 \\ 5.5 & 860 \\ 60 & 2,800 \\ 25 & 1,200\end{array}$

ximu

$$
65
$$

320

2,800

1,200
Depth, in feet

Specific conductance, lab $(\mu \mathrm{S} / \mathrm{cm})$

$\mathrm{pH}$, lab

Sodium (mg/L)

Chloride (mg/L)

Barium $(\mu \mathrm{g} / \mathrm{L})$

Strontium $(\mu \mathrm{g} / \mathrm{L})$ 
shown in figure 3. Although water from 80 (55 percent) of 146 shallow wells was contaminated, water from only 10 (7 percent) of the shallow wells had chloride concentrations greater than $250 \mathrm{mg} / \mathrm{L}$.

The areal extent of brine contamination (chloride concentration equal to or greater than $20 \mathrm{mg} / \mathrm{L}$ ) in deep ground water (depth greater than $120 \mathrm{ft}$ ) in the vicinity of the Brookhaven oil field during August 1993-December 1994 is shown in figure 4. Although water from 25 (81 percent) of 31 deep wells was contaminated, water from only 2 ( 6 percent) of the deep wells had chloride concentrations greater than $250 \mathrm{mg} / \mathrm{L}$.

\section{Change in Brine Contamination from 1981-84 to 1993-94}

During 1993-94, ground-water-quality samples were collected at 81 sites that were sampled during 1981-84. The chloride concentrations for the 1981-84 and 1993-94 study periods and changes in chloride concentrations between the two periods are listed in table 6.

Analyses indicated that between 1981-84 and 1993-94, the chloride concentration increased at some sites and decreased at others due to the movement of brine in the area. Some of the decreases in chloride concentration likely reflect dilution by freshwater recharge by local rainfall. Furthermore, regionally, the total amount of brine in the shallow groundwater system is likely decreasing because of the regional discharge of the shallow ground water to streams. This conclusion is based on reports that significant quantities of brine are no longer being introduced into the groundwater system.

From 1981-84 to $1993-94$, the largest chloride increase $(2,240 \mathrm{mg} / \mathrm{L})$ was at a shallow well (45 ft deep) completed in the Citronelle aquifer (in the western part of the oil field), but the next largest increase $(851 \mathrm{mg} / \mathrm{L})$ was at a moderately deep well (200 ft deep) completed in the Miocene aquifer system (in the eastern part of the oil field).

From 1981-84 to 1993-94, the chloride concentration increased significantly (was greater than $20 \mathrm{mg} / \mathrm{L}$ and increased at least $2.0 \mathrm{mg} / \mathrm{L}$ ) at 23 (28 percent) of 81 sites. All but one well with significant increases in chloride concentration are within the Brookhaven oil field. The one exception (well 3) is located about $2 \mathrm{mi}$ east of the oil field. Only three sections have two or more wells with large (greater than $20 \mathrm{mg} / \mathrm{L}$ ) increases in chloride concentration. Two of these sections, T. 7 N., R. 7 E., sec. 7 and 8, are in the west-central part of the oil field. The other section, T. 8 N., R. 7 E., sec. 33, is in the northeastern part of the oil field.

From 1981-84 to 1993-94, the chloride concentration decreased significantly (was greater than $20 \mathrm{mg} / \mathrm{L}$ and decreased at least $2.0 \mathrm{mg} / \mathrm{L}$ ) at 20 (25 percent) of 81 sites. Most wells with significant decreases in chloride concentration are in the southern two-thirds of the oil field or are southwest of the oil field. Nine wells had large (greater than $20 \mathrm{mg} / \mathrm{L}$ ) decreases in chloride concentration; eight of these wells are in the oil field. The largest 
Table 6. Change in chloride concentrations in ground water in the vicinity of the Brookhaven oil field, Lincoln County, Mississippi (1981-84 to 1993-94) [CRNL, Citronelle aquifer; $M O C N$, Miocene aquifer system; $\mathrm{ft}$, feet; $\mathrm{mg} / \mathrm{L}$, milligrams per liter. Bold data indicate sites with significant chloride changes--at least one chloride concentration exceeded $20 \mathrm{mg} / \mathrm{L}$ and the change was at least $2.0 \mathrm{mg} / \mathrm{L}]$

\begin{tabular}{|c|c|c|c|c|c|c|c|c|}
\hline $\begin{array}{c}\text { Site } \\
\text { number }\end{array}$ & $\begin{array}{l}\text { Local } \\
\text { number }\end{array}$ & $\begin{array}{l}\text { Aquifer } \\
\text { unit } \\
\text { code }\end{array}$ & $\begin{array}{l}\text { Depth } \\
(\mathrm{ft})\end{array}$ & Year & $\begin{array}{l}\text { Chloride } \\
\text { (mg/L) }\end{array}$ & Year & $\begin{array}{l}\text { Chloride } \\
(\mathrm{mg} / \mathrm{L})\end{array}$ & $\begin{array}{l}\text { Chloride } \\
\text { change } \\
\text { (mg/L) }\end{array}$ \\
\hline 3 & C17 & CRNL & 50 & 1983 & 21 & 1994 & 40 & 19 \\
\hline 4 & C18 & CRNL & 65 & 1983 & 42 & 1994 & 34 & -8.0 \\
\hline 5 & C19 & CRNL & 63 & 1983 & 170 & 1993 & 200 & 30 \\
\hline 6 & C20 & CRNL & 100 & 1983 & 4.7 & 1994 & 8.3 & 3.6 \\
\hline 7 & $\mathrm{C} 1$ & CRNL & 40 & 1983 & 7.5 & 1994 & 30 & 22 \\
\hline 8 & $\mathrm{C} 22$ & MOCN & 200 & 1983 & 8.5 & 1994 & 18 & 9.5 \\
\hline 9 & C24 & CRNL & 49 & 1983 & 13 & 1994 & 16 & 3.0 \\
\hline 10 & $\mathbf{C 2 5}$ & CRNL & 60 & 1983 & 57 & 1994 & 29 & -28 \\
\hline 11 & C28 & MOCN & 200 & 1983 & 3.0 & 1994 & 3.0 & .0 \\
\hline 12 & 029 & CRNL & 80 & 1983 & 23 & 1994 & 99 & 76 \\
\hline 13 & C31 & CRNL & 60 & 1983 & 95 & 1994 & 110 & 15 \\
\hline 14 & C32 & CRNL & 100 & 1983 & 7.8 & 1994 & 7.5 & -.3 \\
\hline 15 & $\mathrm{C} 34$ & CRNL & 100 & 1983 & 8.8 & 1994 & 8.4 & -.4 \\
\hline 16 & C35 & CRNL & 70 & 1983 & 100 & 1993 & 140 & 40 \\
\hline 17 & C36 & CRNL & 30 & 1983 & $<.2$ & 1994 & 21 & 21 \\
\hline 18 & C37 & CRNL & 75 & 1983 & 17 & 1994 & 120 & 103 \\
\hline 19 & $\mathrm{C} 45$ & CRNL & 28 & 1984 & 7.9 & 1994 & 3.9 & -4.0 \\
\hline 28 & $\mathrm{~F} 20$ & MOCN & 85 & 1983 & 3.4 & 1994 & 5.7 & 2.3 \\
\hline 29 & F21 & CRNL & 80 & 1983 & 22 & 1994 & 18 & -4.0 \\
\hline 30 & $\mathrm{~F} 24$ & CRNL & 87 & 1983 & 6.0 & 1994 & 6.2 & .2 \\
\hline 31 & $\mathrm{~F} 27$ & CRNL & 98 & 1983 & 9.2 & 1994 & 8.2 & -1.0 \\
\hline 32 & F28 & CRNL & 60 & 1983 & 16 & 1994 & 9.3 & -6.7 \\
\hline 33 & F38 & CRNL & 72 & 1984 & 5.0 & 1994 & 6.7 & 1.7 \\
\hline 34 & F39 & CRNL & 80 & 1984 & 3.5 & 1994 & 3.4 & -.1 \\
\hline 35 & F40 & CRNL & 120 & 1984 & 6.5 & 1994 & 7.1 & .6 \\
\hline 36 & F41 & CRNL & 80 & 1984 & 5.3 & 1994 & 3.6 & -1.7 \\
\hline 37 & F44 & CRNL & 70 & 1983 & 36 & 1994 & 31 & -5.0 \\
\hline 39 & G25 & MOCN & 150 & 1983 & 3.7 & 1993 & 5.5 & 1.8 \\
\hline
\end{tabular}


Table 6. Change in chloride concentrations in ground water in the vicinity of the Brookhaven oil field, Lincoln County, Mississippi (1981-84 to 1993-94)-Continued

\begin{tabular}{|c|c|c|c|c|c|c|c|c|}
\hline $\begin{array}{l}\text { Site } \\
\text { number }\end{array}$ & $\begin{array}{l}\text { Local } \\
\text { number }\end{array}$ & $\begin{array}{l}\text { Aquifer } \\
\text { unit } \\
\text { code }\end{array}$ & $\begin{array}{l}\text { Depth } \\
(f t)\end{array}$ & Year & $\begin{array}{l}\text { Chloride } \\
(\mathrm{mg} / \mathrm{L})\end{array}$ & Year & $\begin{array}{c}\text { Chloride } \\
(\mathrm{mg} / \mathrm{L})\end{array}$ & $\begin{array}{c}\text { Chloride } \\
\text { change } \\
\text { (mg/L) }\end{array}$ \\
\hline 40 & G57 & CRNL & 91 & 1981 & 2.5 & 1993 & 11 & 8.5 \\
\hline 41 & G58 & CRNL & 80 & 1981 & 40 & 1994 & 80 & 40 \\
\hline 42 & G59 & CRNL & 76 & 1981 & 88 & 1994 & 240 & 152 \\
\hline 43 & G60 & CRNL & 100 & 1981 & 120 & 1993 & 400 & 280 \\
\hline 44 & G61 & CRNL & 85 & 1981 & 30 & 1993 & 190 & 160 \\
\hline 45 & G62 & CRNL & 45 & 1981 & 260 & 1994 & 2,500 & 2,240 \\
\hline 46 & G63 & CRNL & 65 & 1981 & 48 & 1994 & 11 & -37 \\
\hline 47 & G66 & CRNL & 48 & 1981 & 50 & 1994 & 33 & -17 \\
\hline 48 & G67 & MOCN & 150 & 1981 & 15 & 1993 & 10 & -5.0 \\
\hline 49 & G68 & MOCN & 308 & 1982 & 20 & 1993 & 58 & 38 \\
\hline 50 & G69 & CRNL & 85 & 1982 & 21 & 1994 & 74 & 53 \\
\hline 51 & G71 & MOCN & 256 & 1981 & 2.7 & 1993 & 3.4 & .7 \\
\hline 52 & G72 & CRNL & 80 & 1983 & 7.8 & 1993 & 8.3 & .5 \\
\hline 53 & G73 & CRNL & 120 & 1983 & 6.5 & 1993 & 5.3 & -1.2 \\
\hline 54 & G74 & MOCN & 120 & 1983 & 3.6 & 1993 & 3.5 & -.1 \\
\hline 55 & G75 & MOCN & 100 & 1983 & 3.7 & 1993 & 3.5 & -.2 \\
\hline 56 & G76 & CRNL & 100 & 1983 & 6.2 & 1993 & 5.2 & -1.0 \\
\hline 57 & G77 & CRNL & 100 & 1983 & 47 & 1993 & 41 & -6.0 \\
\hline 59 & G80 & CRNL & 118 & 1983 & 51 & 1993 & 28 & -23 \\
\hline 60 & G82 & CRNL & 45 & 1983 & 29 & 1993 & 25 & -4.0 \\
\hline 61 & G83 & CRNL & 50 & 1983 & 11 & 1994 & 94 & 83 \\
\hline 62 & G85 & CRNL & 100 & 1983 & 54 & 1993 & 55 & 1.0 \\
\hline 63 & G86 & CRNL & 65 & 1983 & 68 & 1993 & 59 & -9.0 \\
\hline 64 & G87 & CRNL & 60 & 1983 & 9.1 & 1994 & 4.5 & -4.6 \\
\hline 65 & G88 & CRNL & 80 & 1983 & 5.5 & 1993 & 6.6 & 1.1 \\
\hline 66 & G89 & CRNL & 50 & 1983 & 59 & 1993 & 32 & -27 \\
\hline 67 & G91 & CRNL & 106 & 1983 & 44 & 1994 & 5.9 & -38 \\
\hline 68 & G95 & CRNL & 30 & 1983 & 9.5 & 1994 & 5.8 & -3.7 \\
\hline
\end{tabular}


Table 6. Change in chloride concentrations in ground water in the vicinity of the Brookhaven oil field, Lincoln County, Mississippi (1981-84 to 1993-94)-Continued

\begin{tabular}{|c|c|c|c|c|c|c|c|c|}
\hline $\begin{array}{l}\text { Site } \\
\text { number }\end{array}$ & $\begin{array}{l}\text { Local } \\
\text { number }\end{array}$ & $\begin{array}{l}\text { Aquifer } \\
\text { unit } \\
\text { code }\end{array}$ & $\begin{array}{l}\text { Depth } \\
(\mathrm{ft})\end{array}$ & Year & $\begin{array}{l}\text { Chloride } \\
\text { (mg/L) }\end{array}$ & Year & $\begin{array}{l}\text { Chloride } \\
(\mathrm{mg} / \mathrm{L})\end{array}$ & $\begin{array}{c}\text { Chloride } \\
\text { change } \\
\text { (mg/L) }\end{array}$ \\
\hline 69 & G97 & MOCN & 175 & 1983 & 8.0 & 1994 & 6.4 & -1.6 \\
\hline 71 & G100 & CRNL & 90 & 1983 & 49 & 1994 & 74 & 25 \\
\hline 72 & G106 & CRNL & 92 & 1983 & 63 & 1994 & 50 & -13 \\
\hline 73 & G107 & CRNL & 120 & 1983 & 710 & 1993 & 210 & -500 \\
\hline 74 & G109 & CRNL & 79 & 1983 & 56 & 1994 & 66 & 10 \\
\hline 75 & G110 & CRNL & 100 & 1983 & 19 & 1994 & 18 & -1.0 \\
\hline 76 & G114 & CRNL & 84 & 1983 & 210 & 1993 & 590 & 380 \\
\hline 77 & G117 & CRNL & 73 & 1983 & 33 & 1994 & 26 & -7.0 \\
\hline 78 & G118 & CRNL & 69 & 1983 & 120 & 1994 & 39 & -81 \\
\hline 79 & G119 & CRNL & 98 & 1983 & 28 & 1993 & 32 & 4.0 \\
\hline 82 & G127 & CRNL & 70 & 1983 & 130 & 1994 & 180 & 50 \\
\hline 83 & G131 & MOCN & 200 & 1983 & 8.9 & 1994 & 860 & 851 \\
\hline 84 & G133 & CRNL & 120 & 1983 & 6.4 & 1993 & 5.1 & -1.3 \\
\hline 89 & G139 & CRNL & 93 & 1983 & 160 & 1993 & 87 & -73 \\
\hline 90 & G140 & CRNL & 80 & 1983 & 120 & 1993 & 120 & .0 \\
\hline 91 & G142 & CRNL & 40 & 1984 & 3.7 & 1993 & 3.4 & -.3 \\
\hline 92 & G143 & CRNL & 80 & 1984 & 26 & 1993 & 14 & -12 \\
\hline 93 & G144 & CRNL & 75 & 1984 & 9.2 & 1993 & 6.2 & -3.0 \\
\hline 102 & G175 & CRNL & 70 & 1984 & 21 & 1993 & 20 & -1.0 \\
\hline 103 & G177 & CRNL & 120 & 1984 & 3.0 & 1993 & 2.6 & -.4 \\
\hline 104 & G178 & CRNL & 60 & 1984 & 5.1 & 1993 & 4.2 & -.9 \\
\hline 105 & G180 & CRNL & 147 & 1984 & 75 & 1993 & 89 & 14 \\
\hline 106 & G181 & CRNL & 150 & 1984 & 120 & 1993 & 78 & -42 \\
\hline 107 & G182 & CRNL & 148 & 1984 & 3.2 & 1993 & 5.3 & 2.1 \\
\hline 151 & K35 & CRNL & 80 & 1984 & 29 & 1994 & 13 & -16 \\
\hline
\end{tabular}


chloride decrease $(500 \mathrm{mg} / \mathrm{L})$ was in water from a shallow well (120 ft deep) in the southwestern part of the oil field.

\section{SUMMARY}

From August 1993 to December 1994, the U.S. Geological Survey, in cooperation with the U.S. Environmental Protection Agency, collected waterquality data to determine the extent of brine contamination of ground water in the vicinity of the Brookhaven oil field in northwestern Lincoln County, Mississippi. The geologic units of the Citronelle Formation and the Miocene Series that contain sufficient saturated permeable material to yield significant quantities of water serve as aquifers in the study area. These units are identified in this report as the Citronelle aquifer and the Miocene aquifer system. Data indicate that the two aquifers may be hydraulically connected in part of the study area.

Large quantities of brine are withdrawn in conjunction with the production of petroleum. For the last 10 years (1985-94) annual brine production in the Brookhaven oil field has averaged about 550,000 barrels, and the brine-to-oil ratio has averaged about 4.4. From 1943 to 1994, cumulative brine production was about $60,561,000$ barrels. Sodium is the predominant cation (mean concentration, $48,000 \mathrm{mg} / \mathrm{L}$ ) and chloride the predominant anion (mean concentration, $98,500 \mathrm{mg} / \mathrm{L}$ ) in brine samples from the major oil-producing formation (the lower part of the Tuscaloosa Formation) in the Brookhaven oil field.

During 1993-94, sodium concentrations in ground water in the study area ranged from 2.2 to $3,700 \mathrm{mg} / \mathrm{L}$ (median, $14 \mathrm{mg} / \mathrm{L}$ ); chloride, from 2.5 to 9,400 $\mathrm{mg} / \mathrm{L}$ (median, $21 \mathrm{mg} / \mathrm{L}$ ). Water from 89 (50 percent) of 177 wells was considered contaminated by brine--that is, had chloride concentrations greater than $20 \mathrm{mg} / \mathrm{L}$. However, the chloride concentrations in only 12 (7 percent) of 177 wells were greater than the recommended secondary limit for drinking water $(250 \mathrm{mg} / \mathrm{L})$.

From 1981-84 to 1993-94, the chloride concentration increased significantly (was greater than $20 \mathrm{mg} / \mathrm{L}$ and increased at least $2.0 \mathrm{mg} / \mathrm{L}$ ) at 23 sites and decreased significantly (was greater than $20 \mathrm{mg} / \mathrm{L}$ and decreased at least $2.0 \mathrm{mg} / \mathrm{L})$ at 20 sites. The largest chloride increase $(2,240 \mathrm{mg} / \mathrm{L})$ was at a shallow well (45 ft deep) completed in the Citronelle aquifer, but the next largest increase $(851 \mathrm{mg} / \mathrm{L})$ was at a moderately deep well (200 ft deep) completed in the Miocene aquifer system. The largest chloride decrease (500 $\mathrm{mg} / \mathrm{L}$ ) was at a shallow well (45 ft deep). 


\section{REFERENCES}

Bicker, A.R., Jr., 1969, Copiah County geology and mineral resources: Mississippi Department of Natural Resources, Bureau of Geology, $172 \mathrm{p}$.

Boswell, E.H., 1979, The Citronelle aquifers in Mississippi: U.S. Geological Survey Water-Resources Investigations Report 78-131, 1 sheet.

Cross, R.D., and Wales, R.W., 1974, Atlas of Mississippi: Jackson, Mississippi: University Press of Mississippi, $187 \mathrm{p}$.

Fishman, M.J., and Friedman, L.C., eds., 1989, Methods for determination of inorganic substances in water and fluvial sediments ( $3 \mathrm{~d}$ ed.): Techniques of Water-Resources Investigations of the United States Geological Survey, Book 5, Chapter A1, 545 p.

Hawkins, M.E., Jones, O.W., and Pearson, C.A., 1963, Analysis of brines from oil-productive formations in Mississippi and Alabama: U.S. Department of the Interior, Bureau of Mines Report of Investigations 6167, $22 \mathrm{p}$.

Kalkhoff, S.J., 1985, Brine contamination of freshwater aquifers and streams in petroleum producing areas in southern Mississippi: U.S. Geological Survey Water-Resources Investigations Report 85-4117, $106 \mathrm{p}$.

---1986, Brine contamination of shallow ground water and streams in the Brookhaven oil field, Lincoln County, Mississippi: U.S. Geological Survey Water-Resources Investigations Report 86-4087, 57 p.

Mississippi State Oil and Gas Board, 1944-95, Mississippi oil and gas production annual reports, calendar years 1943-94 (published annually).

Newcome, Roy, Jr., 1975, The Miocene aquifer system in Mississippi: U.S. Geological Survey Water-Resources Investigations Report 46-75, 3 sheets.

Newcome, Roy, Jr., and Thomson, F.H., 1970, Water for industrial development in Amite, Franklin, Lincoln, Pike, and Wilkinson Counties, Mississippi: Mississippi Research and Development Center Bulletin, 61 p.

Slack, L.J., and Darden, Daphne, 1991, Summary of aquifer tests in Mississippi, June 1942 through May 1988: U.S. Geological Survey Water-Resources Investigations Report 90-4155, $40 \mathrm{p}$.

Smith, Bruce, Mazzella, Aldo, Heran, William, and Bisdorf, Robert, 1988, Evaluation of airborne geophysical methods to map brine contamination, Brookhaven oil field, Lincoln County, Mississippi: Environmental Monitoring Systems Laboratory, Office of Research and Development, U.S. Environmental Protection Agency, Las Vegas, Nevada, 114 p.

U.S. Environmental Protection Agency, 1986a, Quality criteria for water: U.S. Environmental Protection Agency, EPA--440/5-86-001, variously paginated.

---1986b, Secondary maximum contaminant levels (section 143.3 of part 143, National secondary drinking-water regulations): U.S. Code of Federal Regulations, Title 40, Parts 100 to 149, revised as of July 1, 1986, p. 587-590. 